\title{
Next-to-next-to-leading order spin-orbit effects in the near-zone metric and precession equations of compact binaries
}

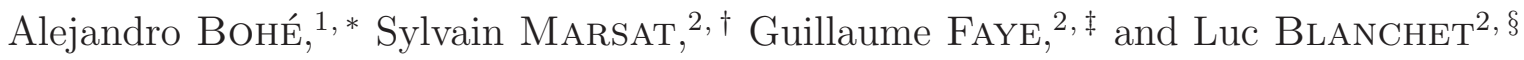 \\ ${ }^{1}$ Departament de Fúsica, Universitat de les Illes Balears, \\ Crta. Valldemossa km 7.5, E-07122 Palma, Spain \\ ${ }^{2} \mathcal{G} \mathbb{R} \varepsilon \mathbb{C O}$ Institut d'Astrophysique de Paris - UMR 7095 du CNRS, \\ Université Pierre \&6 Marie Curie, $98^{\text {bis }}$ boulevard Arago, F-75014 Paris, France
}

(Dated: October 30, 2018)

\begin{abstract}
We extend our previous work devoted to the computation of the next-to-next-to-leading order spin-orbit correction (corresponding to 3.5PN order) in the equations of motion of spinning compact binaries, by: (i) Deriving the corresponding spin-orbit terms in the evolution equations for the spins, the conserved integrals of the motion and the metric regularized at the location of the particles (obtaining also the metric all-over the near zone but with some lower precision); (ii) Performing the orbital reduction of the precession equations, near-zone metric and conserved integrals to the centerof-mass frame and then further assuming quasi-circular orbits (neglecting gravitational radiation reaction). The results are systematically expressed in terms of the spin variables with conserved Euclidean norm instead of the original antisymmetric spin tensors of the pole-dipole formalism. This work paves the way to the future computation of the next-to-next-to-leading order spin-orbit terms in the gravitational-wave phasing of spinning compact binaries.
\end{abstract}

* alejandro.bohe@uib.es

$\dagger$ marsat@iap.fr

$\ddagger$ faye@iap.fr

$\S$ blanchet@iap.fr 


\section{INTRODUCTION}

Coalescing binaries of compact objects - neutron stars and/or black holes — are one of the most promising sources for the detection of gravitational waves (GW) with the advanced versions of the ground-based interferometers LIGO [1, 2] and VIRGO [3, 4], with GEO-HF 5] which will start taking data in the next years, and with the cryogenic detector KAGRA joining the network in a relatively near future [6]. Further ahead, LISA-type space-based interferometers [7, 8], which will significantly increase the accessible region of parameter space, will be able to detect supermassive black-hole binaries with a very high signal-tonoise ratio. Successfully extracting the possibly very weak signal from the noise or estimating the parameters of the source with good accuracy and precision can be achieved by using matched filtering techniques, provided that the waveform is priorly correctly modeled (see e.g. Refs. [9, 10]). The post-Newtonian (PN) approximation scheme enables the computation of precise/accurate templates for the inspiralling phase of the coalescence of compact binaries [11].

For non-spinning objects, both the dynamics of the system and the waveform phase have been derived up to the 3.5PN order 12 14. 1n the last years, an important effort (motivated by astrophysical observations [15-19]) has been undertaken to extend these results to the spinning case, up to the same accuracy. In the present work, we investigate the higher-order binary dynamics focusing on the spin-orbit effects, which are linear in the spin parameter and numerically the most important to be taken into account at leading order. Here by spin, we mean the intrinsic (classical) angular momentum $S$ of the individual compact body, rescaled in our convention by a factor $c$ as $S \equiv c S_{\text {true }}=G m_{\text {body }}^{2} \chi$, where $\chi$ is the dimensionless spin parameter equal to 1 for maximally spinning objects, so that $S$ appears to be formally of Newtonian order and all powers of $1 / c$ are kept explicitly. Adopting this power counting, the leading order spin-orbit and spin-spin contributions to the dynamics appear at $1.5 \mathrm{PN}$ and 2PN respectively [20 25], while the next-to-leading corrections are of 2.5PN [26 31] and $3 \mathrm{PN}$ order [32 36] respectively. Note that the spin-spin couplings between different spins (1 and 2) are actually known at 4PN order [37, 38].

In a recent work [39] 22 we computed the next-to-next-to leading order spin-orbit correction at order 3.5PN in the equations of motion. We used a direct post-Newtonian iteration of the Einstein field equations in harmonic coordinates and proved the equivalence of our result with the one obtained in Ref. [40] using a Hamiltonian approach in ADM coordinates. We have further computed the associated conserved energy, verified the manifest Lorentz invariance of the equations of motion, and found agreement in the test mass limit with the motion of a spinless test particle around a Kerr black hole, as well as that of a spinning test particle around a Schwarzschild black hole. Our calculation was based on the description of the two compact objects as spinning point particles within the framework of the pole-dipole effective model. We refer to Section II of Paper I and references therein for a detailed presentation of the formalism on which the present work also relies. Our purpose here, building on Paper I, is threefold:

1. Deriving the next-to-next-to-leading order spin-orbit corrections to the evolution equations for the spins, the conserved integrals of motion and the metric regularized at the location of the particles. We also obtain an expression for the spin-orbit part of the

\footnotetext{
${ }^{1}$ As usual the $n \mathrm{PN}$ order includes corrections up to the relative order $1 / c^{2 n}$ in a power expansion in the inverse of the speed of light.

${ }^{2}$ Hereafter this work will be referred to as Paper I.
} 
metric valid all over the near-zone up to the highest available order. Knowing explicitly the spin contributions in the near-zone metric is important for applications such as the numerical study of the accretion disk or jet dynamics around spinning back hole binaries [41], and the on-going comparison between PN predictions and the numerical calculation of the self force acting on a particle orbiting a spinning black hole [42, 43].

2. Expressing the results in terms of spin variables with conserved Euclidean norm instead of the original antisymmetric tensors of the pole-dipole formalism as used in Paper I. This choice simplifies the dynamics since the spin evolution equations take the form of ordinary precession equations, and is motivated by our final goal, namely to compute the next-to-next-to-leading spin-orbit effects in the GW orbital phase. The evolution of the GW phase will be indeed obtained via an energy balance argument requiring specifically the use of the conserved-norm spins.

3. Performing the reduction of our results to the center-of-mass frame and to the case of quasi-circular orbits. This is of particular interest for data analysis purposes since, generally, orbits are circularized by emission of gravitational radiation by the time the binary enters the observational band. Notably, we obtain the 3.5PN expression including spin-orbit terms of the binary's energy as a function of the orbital frequency, which is one of the main ingredients entering the construction of the various approximants used for the evolution of the GW phase.

The paper is organized as follows. In Section [II, we present the construction of the conserved-norm spin variable that we use throughout the paper as well as the associated precession equation including the next-to-next-to leading spin-orbit effects, written in a general frame. The other general-frame expressions being too long to be shown here, we directly move on to the presentation of our results in the center-of-mass frame in Section III. The reduction to quasi-circular orbits is performed in Section [V], and the Section V] contains our conclusions. In Appendix $\mathrm{A}$ we compute the correspondence between our spin variables and those of Ref. [40], whereas Appendix B gives the explicit relation between our spin variable and the antisymmetric spin tensor of Paper I. Appendix $\mathrm{C}$ gives the general frame expression of the center-of-mass position at next-to-next-to leading spin-orbit order. We relagate in Appendix D the lengthy expression of the total angular momentum. In Appendix E we show that spin-orbit terms at 3PN order are pure gauge.

Most of our computations were achieved by means of the package xAct, which handles symbolic tensor calculus within the scientific software Mathematica $\AA$ [44].

\section{PRECESSION EQUATIONS OF COMPACT BINARIES}

\section{A. Definition of the constant magnitude spin}

While in Paper I we found most convenient to work with the space components of the spin tensor, in the present article we shall introduce new spin variables, denoted $S_{a}$ (with $a=1,2,3$ ), which are designed to have a conserved Euclidean norm. Using conserved-norm spin vector variables is indeed the most natural choice when considering the dynamics reduced to the center-of-mass frame or to circular orbits. Their evolution equations reduce, by construction, to ordinary precession equations, and these variables are important when 
studying the gravitational waves emitted by a quasi-circular binary because they are secularly constant [45, 46].

For convenience, we change our notations for the spin variables with respect to Paper I. For the spin tensor variable of Paper I we now use the notation:

$$
\tilde{S}^{\mu \nu} \equiv S_{\mathrm{MBFB}}^{\mu \nu}
$$

Let us recall from Section II in Paper I that this variable satisfies the covariant spin supplementary condition (SSC), namely $\tilde{S}^{\mu \nu} p_{\mu}=0$ where $p_{\mu}$ denotes the linear four-momentum of the particle. The covariant spin vector $\widetilde{S}_{\mu}$ associated with the spin tensor is therefore defined by

$$
\tilde{S}^{\mu \nu} \equiv-\frac{1}{\sqrt{-g}} \varepsilon^{\mu \nu \rho \sigma} \frac{p_{\rho}}{m} \tilde{S}_{\sigma},
$$

where $\varepsilon^{\mu \nu \rho \sigma}$ denotes the Levi-Civita symbol; we know that both $p_{\mu} p^{\mu}=-m^{2}$ and $\tilde{S}_{\mu} \tilde{S}^{\mu}=s^{2}$ are conserved along the trajectory: $m=$ const and $s=$ const. Working at linear order in the spins, the linear momentum agrees with the normalized four velocity, $p_{\mu}=m u_{\mu}+\mathcal{O}\left(S^{2}\right)$, and we simply get that:

$$
\frac{\mathrm{D} \tilde{S}_{\mu}}{\mathrm{d} \tau}=\mathcal{O}\left(S^{2}\right),
$$

where $\mathrm{D} / \mathrm{d} \tau \equiv u^{\nu} \nabla_{\nu}$. Thus the spin covector is parallel transported at linear order in spin. We can also impose that the spin should be purely spatial for the comoving observer:

$$
\tilde{S}_{\mu} u^{\mu}=\mathcal{O}\left(S^{2}\right)
$$

From now on, we shall omit writing the $\mathcal{O}\left(S^{2}\right)$ remainders.

A standard, general procedure to define a constant-norm spin vector consists in projecting $\tilde{S}_{\mu}$ onto some orthonormal tetrad $e_{\alpha}{ }^{\mu}$, i.e. a tetrad that satisfies $g_{\mu \nu} e_{\alpha}{ }^{\mu} e_{\beta}{ }^{\nu}=\eta_{\alpha \beta}$, which leads to the four scalar components

$$
S_{\alpha}=e_{\alpha}{ }^{\mu} \tilde{S}_{\mu}
$$

If we choose for the time-like tetrad vector the four-velocity itself, $e_{0}{ }^{\mu}=u^{\mu}$, the time component tetrad projection $S_{0}$ vanishes because of the previous orthogonality condition $\tilde{S}_{\mu} u^{\mu}=0$, and the spatial components $S_{a}$ (with $a=1,2,3$ ) define a constant-norm spin vector. Indeed we have seen that $\tilde{S}_{\mu} \tilde{S}^{\mu}=s^{2}=$ const is conserved along the trajectory. Because $\tilde{S}_{\mu} u^{\mu}=0$ we can rewrite this as $\gamma^{\mu \nu} \tilde{S}_{\mu} \tilde{S}_{\nu}=s^{2}$, in which we have introduced the projector $\gamma^{\mu \nu}=g^{\mu \nu}+u^{\mu} u^{\nu}$ onto the spatial hypersurface orthogonal to $u^{\mu}$. Now, from the orthonormality of the tetrad and from our choice $e_{0}{ }^{\mu}=u^{\mu}$, we have

$$
\gamma^{\mu \nu}=\delta^{a b} e_{a}^{\mu} e_{b}^{\nu}
$$

Therefore the conservation law $\gamma^{\mu \nu} \tilde{S}_{\mu} \tilde{S}_{\nu}=s^{2}$ becomes

$$
\delta^{a b} S_{a} S_{b}=s^{2}
$$

which is the relation defining a Euclidean constant-magnitude spin variable $S_{a}$.

\footnotetext{
${ }^{3}$ For convenience in this Subsection we pose $c=1$.
} 
However, the choice of the spin variable $S_{a}$ is still somewhat arbitrary, since a rotation of the tetrad vectors can always be performed. Here, in order to fix it, we shall achieve a construction equivalent to that of Ref. [29], which presents the advantage of providing a unique determination of these redefined variables in a given gauge. This results in a definition which differs from the one adopted at the previous order in Refs. [28, 46]. In Appendix A we shall study the correspondence between our constant magnitude spin variable and the ADM one, used in Refs. [29, 40].

To uniquely fix the tetrad vectors we proceed in the following way. Consider the spatial covariant components of the projector tensor $\gamma_{\mu \nu}$, namely $\gamma_{i j}=g_{i j}+u_{i} u_{j}$, which as we see from Eq. (2.6) can also be written in terms of space tetrad vectors as $\gamma_{i j}=\delta^{a b} e_{a i} e_{b j}$, where the "mixed-components" tetrad vectors read $e_{a i}=e_{a}{ }^{\mu} \gamma_{i \mu}$. The problem is thus to find a prescription for defining the $3 \times 3$ matrix $e_{a i}$ starting from the $3 \times 3$ matrix $\gamma_{i j}$. Clearly, if $e_{a i}$ is symmetric in its two indices, namely $e_{a i}=e_{i a}$ (in which we are actually exchanging a covariant spatial index $i$ with the Lorentz spatial index $a$ ), then the matrix $e_{a i}$ can be viewed as the square root of the matrix $\gamma_{i j}$. Now, as pointed out in Ref. [29], a positive-definite symmetric matrix such as $\gamma_{i j}$ has a unique symmetric positive-definite square root $e_{a i}$, which therefore satisfies $\gamma_{i j}=\delta^{a b} e_{i a} e_{b j}$. Thanks to this lemmat we have a mean to define the tetrad in a "canonical" way, by adopting for $e_{a i}$ the unique symmetric positive-definite square root of $\gamma_{i j}$. We find

$$
e_{a}^{\mu}=\left(\gamma^{\mu i}-\gamma^{\mu 0} v^{i}\right) e_{a i}
$$

where we have used $e_{a 0}=-v^{i} e_{a i}$ with $v^{i}=u^{i} / u^{0}$ denoting the ordinary coordinate velocity. This, together with $e_{0}{ }^{\mu}=u^{\mu}$, defines completely and uniquely the tetrad and therefore the constant-magnitude spin variable

$$
S_{a}=e_{a}^{\mu} \tilde{S}_{\mu}=\left(e_{a}^{i}-v^{i} e_{a}^{0}\right) \tilde{S}_{i} .
$$

The evolution equation (2.3) for the original spin variable $\tilde{S}_{\mu}$ now translates into an ordinary precession equation for the tetrad components (2.9), namely

$$
\frac{\mathrm{d} S_{a}}{\mathrm{~d} t}=\Omega_{a}^{b} S_{b}
$$

where the precession tensor is given in terms of the (covariant) derivatives of the tetrad by

$$
\Omega_{a}^{b}=-e_{a}{ }^{\mu} \frac{\mathrm{D} e^{b}}{\mathrm{~d} t} .
$$

The antisymmetric character of the matrix $\Omega_{a}{ }^{b}$, which is just made out of the usual Ricci rotation coefficients $\omega^{a}{ }_{\nu}{ }^{b}=-e^{a \mu} \nabla_{\nu} e^{b}{ }_{\mu}=-\omega^{b}{ }_{\nu}{ }^{a}$, namely $\Omega^{a b}=v^{\nu} \omega^{a}{ }_{\nu}{ }^{b}$ where $v^{\nu}=u^{\nu} / u^{0}$, guaranties that $S_{a}$ satisfies an ordinary precession-type equation, i.e.

$$
\frac{\mathrm{d} \mathbf{S}}{\mathrm{d} t}=\Omega \times \mathbf{S}
$$

where we denote $\mathbf{S}=\left(S_{a}\right), \boldsymbol{\Omega}=\left(\Omega_{a}\right)$ and pose $\Omega_{a}=-\frac{1}{2} \varepsilon_{a b c} \Omega^{b c}$. As a consequence of (2.12) the spin has a conserved Euclidean norm, $\mathbf{S}^{2}=s^{2}[c f$ Eq. (2.7)] .

${ }^{4}$ The existence of a symmetric positive-definite square root of a symmetric positive-definite matrix comes from the fact that the latter can be diagonalized and has only positive eigenvalues. The unicity is easily proven after remarking that the original matrix and its square root commute and therefore can be simultaneously diagonalized. 
The latter construction is completely equivalent to the one of Ref. [29]. Indeed the orthonormality condition $g^{\mu \nu} \tilde{S}_{\mu} \tilde{S}_{\nu}=s^{2}$ is rewritten in [29] as $G^{i j} \tilde{S}_{i} \tilde{S}_{j}=s^{2}$, with an effective metric $G^{i j}=g^{i j}-2 g^{0(i} v^{j)}+g^{00} v^{i} v^{j}$, and the conserved norm spin variable is defined there by $S^{i}=H^{i j} \tilde{S}_{j}$, where $H^{i j}$ is the unique symmetric and positive definite square root of the matrix $G^{i j}$. Replacing the metric $g^{\mu \nu}$ by the projector $\gamma^{\mu \nu}=g^{\mu \nu}+u^{\mu} u^{\nu}$, and using $\gamma^{\mu \nu}=\delta^{a b} e_{a}^{\mu} e_{b}{ }^{\nu}$, we obtain the correspondence with our previous tetrad formalism:

$$
G^{i j}=\delta^{a b}\left(e_{a}^{i}-v^{i} e_{a}^{0}\right)\left(e_{b}^{j}-v^{j} e_{b}^{0}\right)
$$

Notice that the effective metric $G^{i j}$ is actually the inverse of the spatial projector $\gamma_{i j}=$ $g_{i j}+u_{i} u_{j}$, i.e. we have $G^{i j} \gamma_{j k}=\delta_{k}^{i}$. From (2.13) we get immediately the correct factorization. Indeed, the matrix $H_{a}{ }^{i}$, such that $G^{i j}=\delta^{k l} H_{k}{ }^{i} H_{l}{ }^{j}$ in the notation of [29], can be written as

$$
H_{a}^{i}=e_{a}^{i}-v^{i} e_{a}^{0}
$$

If we replace the tetrad components by (2.8) in this expression, we find that $H_{a}{ }^{i}=e_{a j} G^{i j}$. Since the symmetric matrices $e_{a i}$ and $G^{i j}$ are, respectively, the square root and the inverse of the matrix $\gamma_{i j}$, we obtain that the matrix $H_{a}{ }^{i}$ is actually the inverse of the matrix $e_{a i}$, namely $H_{a}{ }^{j} e_{b j}=\delta_{a b}$, which shows that it is symmetric and positive definite, as required. As a result, we find that the definition of Ref. [29],

$$
S_{a}=H_{a}{ }^{i} \tilde{S}_{i}
$$

is in fact the same as our definition (2.9). Both procedures explained above are therefore equivalent, but the present approach based on a tetrad is more insightful.

\section{B. Evolution equation for the conserved spin vector}

From now on we shall stick to our definition (2.9) of the constant magnitude spin, and shall no longer make any distinction between the spatial indices $a, b, \cdots$, which were introduced above as tetrad indices, and the ordinary spatial indices $i, j, k, \cdots=1,2,3$, which will be raised and lowered by the Euclidean metric. The 3.5PN gravitational field will be decomposed for convenience in terms of eight metric potentials denoted by $V, V_{i}, \hat{W}_{i j}, \hat{R}_{i}$, $\hat{X}, \hat{Z}_{i j}, \hat{Y}_{i}$ and $\hat{T}$, as introduced in Eqs. (3.1) of Paper I:

$$
\begin{aligned}
g_{00} & =-1+\frac{2}{c^{2}} V-\frac{2}{c^{4}} V^{2}+\frac{8}{c^{6}}\left(\hat{X}+V_{i} V_{i}+\frac{V^{3}}{6}\right) \\
& +\frac{32}{c^{8}}\left(\hat{T}-\frac{1}{2} V \hat{X}+\hat{R}_{i} V_{i}-\frac{1}{2} V V_{i} V_{i}-\frac{V^{4}}{48}\right)+\mathcal{O}\left(\frac{1}{c^{10}}\right), \\
g_{0 i} & =-\frac{4}{c^{3}} V_{i}-\frac{8}{c^{5}} \hat{R}_{i}-\frac{16}{c^{7}}\left(\hat{Y}_{i}+\frac{1}{2} \hat{W}_{i j} V_{j}+\frac{1}{2} V^{2} V_{i}\right)+\mathcal{O}\left(\frac{1}{c^{9}}\right), \\
g_{i j} & =\delta_{i j}\left[1+\frac{2}{c^{2}} V+\frac{2}{c^{4}} V^{2}+\frac{8}{c^{6}}\left(\hat{X}+V_{k} V_{k}+\frac{V^{3}}{6}\right)\right] \\
& +\frac{4}{c^{4}} \hat{W}_{i j}+\frac{16}{c^{6}}\left(\hat{Z}_{i j}+\frac{1}{2} V \hat{W}_{i j}-V_{i} V_{j}\right)+\mathcal{O}\left(\frac{1}{c^{8}}\right) .
\end{aligned}
$$


Each of these potentials is a retarded solution of a flat-space wave equation sourced by components of the stress-energy tensor and appropriate lower order potentials. We refer to Eqs. (3.4) of Paper I for the explicit expression of these source terms.

Our first result is the explicit expression of the precession tensor $\Omega^{i j}$ in terms of the metric at the $3 \mathrm{PN}$ relative order. For a single spinning particle described in the pole-dipole approximation and moving in a fixed background, the evolution equation for the spin is given by Eq. (2.3). The metric and associated Christoffel symbols therein are replaced up to $3 \mathrm{PN}$ order by the potentials $V, \cdots, \hat{Y}_{i}$, and the spin variable is changed to conform with the definition (2.9) so that we can read off the precession tensor from Eq. (2.10). In practice we use the following explicit expression in terms of the matrix $H^{i j}$ defined by Eq. (2.14):

$$
\Omega^{i j}=\frac{\mathrm{d} H^{i k}}{\mathrm{~d} t}\left(H^{-1}\right)^{k j}+H^{i k} K^{k l}\left(H^{-1}\right)^{l j}
$$

where the matrix $K^{i j}$ is a combination of velocities and Christoffel symbols:

$$
K^{i j}=c \Gamma_{0 i}^{j}+v^{k} \Gamma_{i k}^{j}-\left(\Gamma_{0 i}^{0}+\frac{v^{k}}{c} \Gamma_{i k}^{0}\right) v^{j}
$$

We then obtain the anti-symmetric precession tensor up to 3PN order as

$$
\begin{aligned}
\Omega^{i j}= & \frac{1}{c^{2}}\left[-4 \partial^{[i} V^{j]}-3 v^{[i} \partial^{j]} V\right]+\frac{1}{c^{4}}\left[-8 \partial^{[i} \hat{R}^{j]}+8 V \partial^{[i} V^{j]}+4 v^{k} \partial^{[i} \hat{W}^{j]}{ }_{k}-\frac{1}{4} v^{2} v^{[i} \partial^{j]} V\right. \\
& \left.+4 V^{[i} \partial^{j]} V+2 v^{k} v^{[i} \partial^{j]} V_{k}+2 v^{k} v^{[i} \partial_{k} V^{j]}\right]+\frac{1}{c^{6}}\left[16 V \partial^{[i} \hat{R}^{j]}-16 V^{2} \partial^{[i} V^{j]}-16 v^{k} V_{k} \partial^{[i} V^{j]}\right. \\
& -8 V_{k} \partial^{[i} \hat{W}^{j]}{ }_{k}-16 \partial^{[i} \hat{Y}^{j]}+16 v^{k} \partial^{[i} \hat{Z}^{j]}{ }_{k}+4 v^{k} v^{[i} \partial^{j]} \hat{R}_{k}+8 \hat{R}^{[i} \partial^{j]} V-V v^{2} v^{[i} \partial^{j]} V \\
& -\frac{1}{8} v^{4} v^{[i} \partial^{j]} V+8 V V^{[i} \partial^{j]} V+v^{2} V^{[i} \partial^{j]} V-2 v^{k} V_{k} v^{[i} \partial^{j]} V-5 v^{k} \hat{W}^{[i}{ }_{k} \partial^{j]} V+4 V v^{k} v^{[i} \partial^{j]} V_{k} \\
& +\frac{1}{2} v^{2} v^{k} v^{[i} \partial^{j]} V_{k}+8 v^{k} V^{[i} \partial^{j]} V_{k}-16 V_{k} v^{[i} \partial^{j]} V_{k}-12 v^{[i} \partial^{j]} \hat{X}+4 v^{k} v^{[i} \partial_{k} \hat{R}^{j]} \\
& -4 v^{k} v^{[i} V^{j]} \partial_{k} V+5 v^{[i} \hat{W}^{j]} \partial_{k} V+4 V v^{k} v^{[i} \partial_{k} V^{j]}+\frac{1}{2} v^{2} v^{k} v^{[i} \partial_{k} V^{j]}-8 v^{k} V^{[i} \partial_{k} V^{j]} \\
& \left.-8 V_{k} v^{[i} \partial_{k} V^{j]}+8 \hat{W}^{[i}{ }_{k} \partial_{k} V^{j]}-v^{k} v^{l} v^{[i} \partial_{l} \hat{W}^{j]}{ }_{k}+v^{k} v^{[i} \partial_{t} \hat{W}^{j]}{ }_{k}\right] .
\end{aligned}
$$

This quantity is to be evaluated at the location of the considered particle, at which point all the potentials $V, V_{i}, \cdots$, that parametrize the metric are diverging. For a system of $N$ particles, the metric is the one generated by the stress-energy tensor of the system of the $N$ particles itself. We consider the case $N=2$ and compute the precession tensor (2.19) at the location of, say, particle 1 , the velocity appearing in (2.19) being thus the ordinary velocity of particle 1 .

The evaluation of (2.19) at 1, i.e. at point $\mathbf{y}_{1}$, is made meaningful through Hadamard's regularization [47], consistently with our computation of the 3.5PN spin-orbit acceleration in Paper I. It must be understood in Eq. (2.17) that each term is regularized before taking the products and the time derivative. Nonetheless, although Hadamard's regularization is "non-distributive" in the sense that $(F G)_{1} \neq(F)_{1}(G)_{1}$ in general, we checked that, at the order considered here, treating it as "distributive" makes no difference. Note that the time 
derivative operation does not commute with the regularization operation at 1 , and we have generically for singular functions $F$ in the class considered in Ref. [47] 5

$$
\frac{\mathrm{d}}{\mathrm{d} t}(F)_{1}=\left(\partial_{t} F\right)_{1}+\left(v_{1}^{i} \partial_{i} F\right)_{1}
$$

where $(G)_{1}$ represents the value of $G$ at particle 1 position in the sense of the Hadamard partie finie. In order to present a closed-form expression for $\Omega^{i j}$ in terms of the metric potentials, we first applied the total time derivative there according to the Leibniz rule on individual monomials composing $H^{i j}$, applying the distributivity ansatz $\left[\right.$ i.e. $\left.(F G)_{1}=(F)_{1}(G)_{1}\right]$ for the products. We next replaced the accelerations by their expressions in terms of the potentials. For the time derivatives of quantities regularized at 1, we resorted to Eq. (2.20). Finally, the partial time derivatives of the potentials were eliminated in turn by means of the identities (3.28) of Ref. [48], which are equivalent to the harmonic gauge condition.

Since we are working at linear order in the spins, only the non-spin parts of the metric potentials enter the computation of the matrix $\Omega^{i j}$. Most of those contributions are the same as those required for the $2 \mathrm{PN}$ equations of motion without spin. 6 There are only two genuine 3PN potentials: One of them, $\hat{Z}_{i j}$ at Newtonian order, has the same structure as $\hat{W}_{i j}$; The other one, $\hat{Y}_{i}$, which enters the term $-16 \partial^{[i} \hat{Y}^{j]}$ in Eq. (2.19), shows a higher order of non-linearity (in powers of $G$ ). Only its regularized value can be computed, using dimensional regularization in principle, as was done for the 3PN equations of motion without spin obtained in [49]. Like for the term $\tilde{S}^{j k}\left(\partial_{i j} \hat{Y}_{k}\right)_{1}$ appearing in the equations of motion (see Section V of Paper I), we find that the corrections coming from the dimensional regularization exactly cancel out because of the antisymmetrization due to the contraction with the spin tensor. Thus, like in Paper I, Hadamard's regularization is sufficient for our purpose here. The remaining $3 \mathrm{PN}$ metric potential, $\hat{T}$, does not contribute.

Due to the length of the expression, we relegate to Appendix B the relation between the conserved spin vector and the spin tensor in terms of the orbital variables derived from Eqs. (2.2) and (2.9). We conclude this Section by giving the explicit expression for the precession equation of the conserved spin 1:

$$
\frac{\mathrm{d} \mathbf{S}_{1}}{\mathrm{~d} t}=\mathbf{\Omega}_{1} \times \mathbf{S}_{1}
$$

The vector $\Omega_{1}$ may be expanded at $3 \mathrm{PN}$ order in the form:

$$
\Omega_{1}=\frac{1}{c^{2}} \Omega_{1}^{1 \mathrm{PN}}+\frac{1}{c^{4}} \Omega_{1}^{2 \mathrm{PN}}+\frac{1}{c^{6}} \Omega_{1}^{3 \mathrm{PN}}+\mathcal{O}\left(\frac{1}{c^{7}}\right) .
$$

Except for the spin tensor, we use the same notations for the orbital variables as in Paper I: ( $u v$ ) denotes the scalar product $\mathbf{u} \cdot \mathbf{v}=u^{i} v^{i}$ and $\mathbf{w}=\mathbf{u} \times \mathbf{v}$ the cross product between $\mathbf{u}$ and $\mathbf{v}$, whose components are given by $w^{i}=\varepsilon^{i j k} u^{j} v^{k}$. At leading order, we have

$$
\Omega_{1}^{1 \mathrm{PN}}=\frac{G}{r_{12}^{2}} m_{2}\left[\frac{3}{2} \mathbf{n}_{12} \times \mathbf{v}_{1}-2 \mathbf{n}_{12} \times \mathbf{v}_{2}\right],
$$

\footnotetext{
5 This equation states that, formally, the Hadamard regularization commutes with the operator $v_{1}^{\mu} \partial_{\mu}$.

${ }^{6}$ The non-spin part of the acceleration has the form $a^{i}=F^{i}-\mathrm{d} Q^{i} / \mathrm{d} t$ with $Q^{i}=P^{i}-v^{i}$; see Eqs. (3.5) and Eqs. (3.7) in Paper I.
} 
while the next-to-leading order correction is given by

$$
\boldsymbol{\Omega}_{1}^{2 \mathrm{PN}}=\frac{G}{r_{12}^{2}}{ }^{(2)} \boldsymbol{\Omega}_{1}^{0,1} m_{2}+\frac{G^{2}}{r_{12}^{3}}\left[{ }^{(2)} \boldsymbol{\Omega}_{1}^{0,2} m_{2}^{2}+{ }^{(2)} \boldsymbol{\Omega}_{1}^{1,1} m_{1} m_{2}\right],
$$

where

$$
\begin{aligned}
{ }^{(2)} \mathbf{\Omega}_{1}^{0,1} & =\mathbf{n}_{12} \times \mathbf{v}_{1}\left[-\frac{9}{4}\left(n_{12} v_{2}\right)^{2}+\frac{1}{8} v_{1}^{2}-\left(v_{1} v_{2}\right)+v_{2}^{2}\right]+\mathbf{v}_{1} \times \mathbf{v}_{2}\left[\left(n_{12} v_{1}\right)-\frac{3}{2}\left(n_{12} v_{2}\right)\right] \\
& +\mathbf{n}_{12} \times \mathbf{v}_{2}\left[3\left(n_{12} v_{2}\right)^{2}+2\left(v_{1} v_{2}\right)-2 v_{2}^{2}\right] \\
{ }^{(2)} \mathbf{\Omega}_{1}^{1,1} & =\frac{3}{2} \mathbf{n}_{12} \times \mathbf{v}_{1}+\mathbf{n}_{12} \times \mathbf{v}_{2} \\
{ }^{(2)} \mathbf{\Omega}_{1}^{0,2} & =-\frac{1}{2} \mathbf{n}_{12} \times \mathbf{v}_{1}+\frac{5}{2} \mathbf{n}_{12} \times \mathbf{v}_{2} .
\end{aligned}
$$

These results can either be directly computed from Eq. (2.19) truncated to the appropriate order or obtained by a mere translation of the spin evolution equation given in Section VI of Paper I using the relation (B1) of Appendix B. Using Eq. (2.19), we get the next-to-nextto-leading order correction, new with this paper, to the precession vector:

$$
\begin{aligned}
\Omega_{1}^{3 \mathrm{PN}}= & \frac{G}{r_{12}^{2}}{ }^{(3)} \boldsymbol{\Omega}_{1}^{0,1} m_{2}+\frac{G^{2}}{r_{12}^{3}}\left[{ }^{(3)} \boldsymbol{\Omega}_{1}^{0,2} m_{2}^{2}+{ }^{(3)} \boldsymbol{\Omega}_{1}^{1,1} m_{1} m_{2}\right] \\
& +\frac{G^{3}}{r_{12}^{4}}\left[{ }^{(3)} \boldsymbol{\Omega}_{1}^{0,3} m_{2}^{3}+{ }^{(3)} \boldsymbol{\Omega}_{1}^{1,2} m_{1} m_{2}^{2}+{ }^{(3)} \boldsymbol{\Omega}_{1}^{2,1} m_{1}^{2} m_{2}\right],
\end{aligned}
$$

where

$$
\begin{aligned}
{ }^{(3)} \mathbf{\Omega}_{1}^{0,1}= & \mathbf{n}_{12} \times \mathbf{v}_{1}\left[\frac{45}{16}\left(n_{12} v_{2}\right)^{4}-\frac{3}{16}\left(n_{12} v_{2}\right)^{2} v_{1}^{2}+\frac{1}{16} v_{1}^{4}+\frac{3}{2}\left(n_{12} v_{2}\right)^{2}\left(v_{1} v_{2}\right)-\frac{1}{4} v_{1}^{2}\left(v_{1} v_{2}\right)+v_{2}^{4}\right. \\
& \left.-\frac{15}{4}\left(n_{12} v_{2}\right)^{2} v_{2}^{2}+\frac{1}{4} v_{1}^{2} v_{2}^{2}-\left(v_{1} v_{2}\right) v_{2}^{2}\right]+\mathbf{n}_{12} \times \mathbf{v}_{2}\left[-\frac{15}{4}\left(n_{12} v_{2}\right)^{4}+6\left(n_{12} v_{2}\right)^{2} v_{2}^{2}-2 v_{2}^{4}\right. \\
& \left.-3\left(n_{12} v_{2}\right)^{2}\left(v_{1} v_{2}\right)+2\left(v_{1} v_{2}\right) v_{2}^{2}\right]+\mathbf{v}_{1} \times \mathbf{v}_{2}\left[\frac{1}{4}\left(n_{12} v_{1}\right) v_{1}^{2}+\frac{9}{4}\left(n_{12} v_{2}\right)^{3}+\left(n_{12} v_{1}\right) v_{2}^{2}\right. \\
& \left.-\frac{3}{2}\left(n_{12} v_{1}\right)\left(n_{12} v_{2}\right)^{2}-\frac{1}{8}\left(n_{12} v_{2}\right) v_{1}^{2}-\frac{1}{2}\left(n_{12} v_{1}\right)\left(v_{1} v_{2}\right)+\frac{3}{2}\left(n_{12} v_{2}\right)\left(v_{1} v_{2}\right)-\frac{5}{2}\left(n_{12} v_{2}\right) v_{2}^{2}\right], \\
{ }^{(3)} \mathbf{\Omega}_{1}^{1,1}= & \mathbf{n}_{12} \times \mathbf{v}_{1}\left[-\frac{27}{2}\left(n_{12} v_{1}\right)^{2}+27\left(n_{12} v_{1}\right)\left(n_{12} v_{2}\right)-14\left(n_{12} v_{2}\right)^{2}+\frac{29}{4} v_{1}^{2}-\frac{67}{4}\left(v_{1} v_{2}\right)\right. \\
& \left.+\frac{71}{8} v_{2}^{2}\right]+\mathbf{n}_{12} \times \mathbf{v}_{2}\left[\frac{109}{4}\left(n_{12} v_{1}\right)^{2}-\frac{101}{2}\left(n_{12} v_{1}\right)\left(n_{12} v_{2}\right)+\frac{73}{4}\left(n_{12} v_{2}\right)^{2}-\frac{37}{4} v_{1}^{2}\right. \\
& \left.+\frac{35}{2}\left(v_{1} v_{2}\right)-\frac{33}{4} v_{2}^{2}\right]+\mathbf{v}_{1} \times \mathbf{v}_{2}\left[-\frac{71}{8}\left(n_{12} v_{1}\right)+\frac{55}{8}\left(n_{12} v_{2}\right)\right], \\
{ }_{(3)} \mathbf{\Omega}_{1}^{0,2}= & \mathbf{n}_{12} \times \mathbf{v}_{1}\left[\frac{1}{2}\left(n_{12} v_{1}\right)^{2}-\frac{3}{2}\left(n_{12} v_{1}\right)\left(n_{12} v_{2}\right)+2\left(n_{12} v_{2}\right)^{2}+\frac{3}{8} v_{1}^{2}-\frac{3}{8}\left(v_{1} v_{2}\right)-\frac{1}{2} v_{2}^{2}\right] \\
+ & \mathbf{n}_{12} \times \mathbf{v}_{2}\left[-5\left(n_{12} v_{2}\right)^{2}-\frac{1}{2} v_{1}^{2}-2\left(v_{1} v_{2}\right)+\frac{5}{2} v_{2}^{2}\right]+\mathbf{v}_{1} \times \mathbf{v}_{2}\left[-\frac{5}{8}\left(n_{12} v_{1}\right)-\frac{1}{2}\left(n_{12} v_{2}\right)\right]
\end{aligned}
$$




$$
\begin{aligned}
& { }^{(3)} \boldsymbol{\Omega}_{1}^{2,1}=-\frac{69}{8} \mathbf{n}_{12} \times \mathbf{v}_{1}+\frac{3}{2} \mathbf{n}_{12} \times \mathbf{v}_{2} \\
& { }^{(3)} \boldsymbol{\Omega}_{1}^{1,2}=-\frac{31}{4} \mathbf{n}_{12} \times \mathbf{v}_{1}-\frac{17}{2} \mathbf{n}_{12} \times \mathbf{v}_{2}, \\
& { }^{(3)} \boldsymbol{\Omega}_{1}^{0,3}=\frac{7}{8} \mathbf{n}_{12} \times \mathbf{v}_{1}-\frac{11}{2} \mathbf{n}_{12} \times \mathbf{v}_{2} .
\end{aligned}
$$

\section{COMPACT BINARIES IN THE CENTER-OF-MASS FRAME}

\section{A. Reduction to the center-of-mass frame}

In this Section, we present our results in the center-of-mass (CM) frame, defined by the nullity of the center-of-mass position $\mathrm{G}$. We remind that the latter vector is directly linked to the conserved integral of the motion $\mathbf{K}$ associated with the boost invariance of the conservative part of the dynamics, through the relation $\mathbf{K}=\mathbf{G}-\mathbf{P} t$, with $\mathbf{P}$ being the total linear momentum of the binary. We shall display $\mathbf{G}$ in a general frame at the 3.5PN order in Appendix C. However, it turns out that only the $2.5 \mathrm{PN}$ order is needed to reduce the results to the $\mathrm{CM}$ frame, since $3.5 \mathrm{PN}$ corrections that would matter for the reduction of the Newtonian part can be seen to cancel out based on symmetry arguments, as detailed at the end of Appendix C. When working in the CM frame, it is convenient to use the standard mass parameters $m \equiv m_{1}+m_{2}, \delta m \equiv m_{1}-m_{2}$ and $\nu \equiv m_{1} m_{2} / m^{2}$ (such that $0<\nu \leq 1 / 4$ ), and to introduce the same combinations of spin variables as in Refs. [28, 46], namely

$$
\begin{aligned}
\mathbf{S} & \equiv \mathbf{S}_{1}+\mathbf{S}_{2}, \\
\mathbf{\Sigma} & \equiv m\left(\frac{\mathbf{S}_{2}}{m_{2}}-\frac{\mathbf{S}_{1}}{m_{1}}\right) .
\end{aligned}
$$

Notice that since we are working with different definitions of $\mathbf{S}_{1}$ and $\mathbf{S}_{2}$, our variables $\mathbf{S}$ and $\boldsymbol{\Sigma}$ also differ from those used in Refs. [28, 46]; however, [46] introduced in their Section VII conserved-norm spin variables, which turn out to be identical to ours at 1PN order in the center-of-mass frame. Neither $\mathbf{S}$ nor $\boldsymbol{\Sigma}$ are of conserved norm and, therefore, their evolution equations cannot reduce to precession equations; they can straightforwardly be written in terms of the precession vectors $\Omega_{1}$ and $\Omega_{2}$ as

$$
\begin{aligned}
\frac{\mathrm{d} \mathbf{S}}{\mathrm{d} t} & =\left(\frac{m_{1}}{m} \boldsymbol{\Omega}_{1}+\frac{m_{2}}{m} \boldsymbol{\Omega}_{2}\right) \times \mathbf{S}+\nu\left(\boldsymbol{\Omega}_{\mathbf{2}}-\boldsymbol{\Omega}_{\mathbf{1}}\right) \times \boldsymbol{\Sigma}, \\
\frac{\mathrm{d} \boldsymbol{\Sigma}}{\mathrm{d} t} & =\left(\frac{m_{2}}{m} \boldsymbol{\Omega}_{1}+\frac{m_{1}}{m} \boldsymbol{\Omega}_{2}\right) \times \boldsymbol{\Sigma}+\left(\boldsymbol{\Omega}_{\mathbf{2}}-\boldsymbol{\Omega}_{\mathbf{1}}\right) \times \mathbf{S} .
\end{aligned}
$$

Here, we shall simply give the CM-frame expression for $\Omega_{1}$.

The vector $\mathbf{G}$ including spin-orbit effects at $2.5 \mathrm{PN}$ order was computed in Ref. [28]. Translating it to our spin variables and imposing $\mathbf{G}=\mathbf{0}$, we find the following relations between the positions $\mathbf{y}_{1}, \mathbf{y}_{2}$ in the CM frame and the relative position $\mathbf{x}=\mathbf{y}_{1}-\mathbf{y}_{2}$ and velocity $\mathbf{v}=\mathrm{d} \mathbf{x} / \mathrm{d} t=\mathbf{v}_{1}-\mathbf{v}_{2}=\mathbf{v}_{12}$ (as well as spin variables). Posing

$$
\mathbf{y}_{1}=\frac{m_{2}}{m} \mathbf{x}+\mathbf{z}
$$




$$
\mathbf{y}_{2}=-\frac{m_{1}}{m} \mathbf{x}+\mathbf{z}
$$

we obtain

$$
\begin{aligned}
\mathbf{z}= & \frac{\nu}{2 c^{2}} \frac{\delta m}{m}\left\{v^{2}-\frac{G m}{r}\right\} \mathbf{x}-\frac{\nu}{m c^{3}} \mathbf{\Sigma} \times \mathbf{v}+\frac{r \nu}{c^{4}} \frac{\delta m}{m}\left\{\left(\frac{3}{8}-\frac{3}{2} \nu\right) v^{4} \mathbf{n}\right. \\
+ & \left.\frac{G m}{r}\left\{\left(\left(-\frac{1}{8}+\frac{3}{4} \nu\right)(n v)^{2}+\left(\frac{19}{8}+\frac{3}{2} \nu\right) v^{2}\right) \mathbf{n}-\frac{7}{4}(n v) \mathbf{v}\right]+\frac{G^{2} m^{2}}{r^{2}}\left(\frac{7}{4}-\frac{\nu}{2}\right) \mathbf{n}\right\} \\
+ & \frac{\nu}{m c^{5}}\left\{\left(-\frac{1}{2}+2 \nu\right) v^{2} \mathbf{\Sigma} \times \mathbf{v}+\frac{G m}{r}\left(\frac{\delta m}{m}\left[(n, S, v) \mathbf{n}-\frac{3}{2}(n v) \mathbf{n} \times \mathbf{S}-\frac{1}{2} \mathbf{S} \times \mathbf{v}\right]\right.\right. \\
& +(-1+4 \nu)(n v) \mathbf{n} \times \mathbf{\Sigma}-(2+\nu) \mathbf{\Sigma} \times \mathbf{v})\}+\mathcal{O}\left(\frac{1}{c^{6}}\right) .
\end{aligned}
$$

Note that the explicit relation between the center of mass velocities $\mathbf{v}_{1}, \mathbf{v}_{2}$ and the relative variables $\mathbf{v}, \mathbf{x}$, as well as the two spins, can be obtained either by taking the time derivatives of Eqs. (3.3) and using the evolution equations, or equivalently by imposing that the explicit expression of the total linear momentum $\mathbf{P}$ up to $2.5 \mathrm{PN}$ order satisfies $\mathbf{P}=\mathbf{0}$, which must hold in the center of mass frame, at that approximation level.

For each quantity of interest, we only present the (linear in) spin part, which already leads to somewhat lengthy expressions, and refer to the literature for the non-spin terms. However, one should keep in mind that the spin part of a quantity in the CM frame is not the reduction to the $\mathrm{CM}$ frame of the spin part of the same quantity expressed in a general frame, because of the crucial additional spin contributions due to the replacement of Eqs. (3.3) into the non-spin part.

\section{B. Precession equation}

In the center of mass frame, the expression for the $3 \mathrm{PN}$ precession vector $\Omega_{1}$ defined by Eq. (2.22) reduces to

$$
\begin{aligned}
& \Omega_{1}=\mathbf{n} \times \mathbf{v}\left[\frac{1}{c^{2}} \alpha_{1 \mathrm{PN}}+\frac{1}{c^{4}} \alpha_{2 \mathrm{PN}}+\frac{1}{c^{6}} \alpha_{3 \mathrm{PN}}+\mathcal{O}\left(\frac{1}{c^{7}}\right)\right] \\
& \alpha_{1 \mathrm{PN}}=\frac{G m}{r^{2}}\left(\frac{3}{4}+\frac{1}{2} \nu-\frac{3}{4} \frac{\delta m}{m}\right), \\
& \alpha_{2 \mathrm{PN}=} \frac{G m}{r^{2}}\left[\left(-\frac{3}{2} \nu+\frac{3}{4} \nu^{2}-\frac{3}{2} \nu \frac{\delta m}{m}\right)(n v)^{2}+\left(\frac{1}{16}+\frac{11}{8} \nu-\frac{3}{8} \nu^{2}+\frac{\delta m}{m}\left(-\frac{1}{16}+\frac{1}{2} \nu\right)\right) v^{2}\right] \\
&+\frac{G^{2} m^{2}}{r^{3}}\left(-\frac{1}{4}-\frac{3}{8} \nu+\frac{1}{2} \nu^{2}+\frac{\delta m}{m}\left(\frac{1}{4}-\frac{1}{8} \nu\right)\right), \\
& \alpha_{3 \mathrm{PN}}= \frac{G m}{r^{2}}\left[\left(\frac{15}{8} \nu-\frac{195}{32} \nu^{2}+\frac{15}{16} \nu^{3}+\frac{\delta m}{m}\left(\frac{15}{8} \nu-\frac{75}{32} \nu^{2}\right)\right)(n v)^{4}\right.
\end{aligned}
$$




$$
\begin{aligned}
+ & \left(-3 \nu+\frac{291}{32} \nu^{2}-\frac{45}{16} \nu^{3}+\frac{\delta m}{m}\left(-3 \nu+\frac{177}{32} \nu^{2}\right)\right)(n v)^{2} v^{2} \\
+ & \left.\left(\frac{1}{32}+\frac{19}{16} \nu-\frac{31}{8} \nu^{2}+\frac{17}{16} \nu^{3}+\frac{\delta m}{m}\left(-\frac{1}{32}+\frac{3}{4} \nu-\frac{11}{8} \nu^{2}\right)\right) v^{4}\right] \\
+\frac{G^{2} m^{2}}{r^{3}} & {\left[\left(\frac{1}{4}-\frac{525}{32} \nu-\frac{159}{16} \nu^{2}+\frac{13}{4} \nu^{3}+\frac{\delta m}{m}\left(-\frac{1}{4}-\frac{75}{32} \nu-\frac{87}{16} \nu^{2}\right)\right)(n v)^{2}\right.} \\
& \left.+\left(\frac{3}{16}+\frac{27}{4} \nu+\frac{75}{32} \nu^{2}-\frac{9}{8} \nu^{3}+\frac{\delta m}{m}\left(-\frac{3}{16}+\frac{9}{8} \nu+\frac{35}{32} \nu^{2}\right)\right) v^{2}\right] \\
+\frac{G^{3} m^{3}}{r^{4}} & \left(\frac{7}{16}-\frac{9}{4} \nu-\frac{9}{8} \nu^{2}+\frac{1}{2} \nu^{3}+\frac{\delta m}{m}\left(-\frac{7}{16}-\frac{1}{8} \nu-\frac{1}{8} \nu^{2}\right)\right) .
\end{aligned}
$$

We obtain $\Omega_{2}$ from $\Omega_{1}$ simply by performing the substitution $\delta m \rightarrow-\delta m$.

\section{Relative acceleration}

The acceleration including spin-orbit effects up to 3.5PN order was obtained in Paper I. The reduction to the CM frame yields

$$
\begin{aligned}
& \frac{\mathrm{d} \mathbf{v}}{\mathrm{d} t}=\mathbf{B}_{\mathrm{N}}+\frac{1}{c^{2}} \mathbf{B}_{1 \mathrm{PN}}+\frac{1}{c^{3}} \underset{S}{\mathbf{B}_{1.5 \mathrm{PN}}}+\frac{1}{c^{4}}\left[\mathbf{B}_{2 \mathrm{PN}}+\underset{S S}{\mathbf{B}_{2} \mathrm{PN}}\right]+\frac{1}{c^{5}}\left[\mathbf{B}_{2.5 \mathrm{PN}}+\underset{S}{\mathbf{B}_{2.5 \mathrm{PN}}}\right]
\end{aligned}
$$

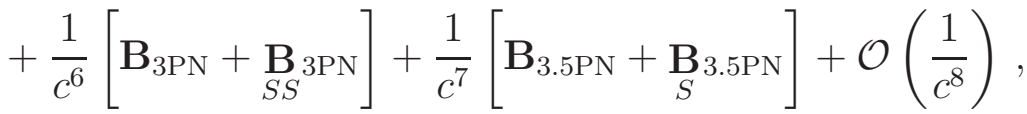

where we have indicated the contributions of spin-spin terms (not considered in the present work); the non-spin terms can be found in Ref. [11]. In the rest of this paper, we use the notation $(u, v, w)$ for the mixed product $\varepsilon^{i j k} u^{i} v^{j} w^{k}$. For the spin-orbit terms, we have at leading order the standard result [22, 23]

$$
\begin{aligned}
m \mathbf{B}_{S} 1.5 \mathrm{PN}=\frac{G m}{r^{3}} & {\left[-6 \frac{\delta m}{m}(n, \Sigma, v) \mathbf{n}-12(n, S, v) \mathbf{n}+9(n v) \mathbf{n} \times \mathbf{S}\right.} \\
& \left.+3 \frac{\delta m}{m}(n v) \mathbf{n} \times \mathbf{\Sigma}+7 \mathbf{S} \times \mathbf{v}+3 \frac{\delta m}{m} \mathbf{\Sigma} \times \mathbf{v}\right] .
\end{aligned}
$$

At next-to-leading order, the results of [28] may be rewritten, with our choice of spin variables, as

$$
m \underset{S}{\mathbf{B}_{2.5 \mathrm{PN}}}=\frac{G m}{r^{3}}\left[{ }^{(2.5)} \mathbf{b}_{1}+{ }^{(2.5)} \mathbf{b}_{2} \frac{G m}{r}\right]
$$

where

$$
\begin{aligned}
{ }^{(2.5)} \mathbf{b}_{1} & =\mathbf{n} \times \mathbf{S}\left[-\frac{45}{2} \nu(n v)^{3}+\left(-\frac{3}{2}+\frac{45}{2} \nu\right)(n v) v^{2}\right]+(n, \Sigma, v) \mathbf{v} \frac{\delta m}{m}\left(\frac{9}{2}-6 \nu\right)(n v) \\
& +\mathbf{n} \times \mathbf{\Sigma}\left[-15 \nu \frac{\delta m}{m}(n v)^{3}+\frac{\delta m}{m}\left(-\frac{3}{2}+12 \nu\right)(n v) v^{2}\right]+(n, S, v) \mathbf{v}\left(\frac{21}{2}-\frac{21}{2} \nu\right)(n v) \\
& +\mathbf{S} \times \mathbf{v}\left[\left(-\frac{3}{2}-15 \nu\right)(n v)^{2}+14 \nu v^{2}\right]+\boldsymbol{\Sigma} \times \mathbf{v}\left[\frac{\delta m}{m}\left(-\frac{3}{2}-9 \nu\right)(n v)^{2}+7 \nu \frac{\delta m}{m} v^{2}\right]
\end{aligned}
$$




$$
\begin{aligned}
& +(n, \Sigma, v) \mathbf{n}\left[15 \nu \frac{\delta m}{m}(n v)^{2}-12 \nu \frac{\delta m}{m} v^{2}\right]+(n, S, v) \mathbf{n}\left[30 \nu(n v)^{2}-24 \nu v^{2}\right], \\
{ }^{(2.5)} \mathbf{b}_{2} & =(n, \Sigma, v) \mathbf{n} \frac{\delta m}{m}\left(24+\frac{37}{2} \nu\right)+(n, S, v) \mathbf{n}(44+33 \nu)+\mathbf{n} \times \mathbf{S}(-28-29 \nu)(n v) \\
& +\mathbf{n} \times \mathbf{\Sigma} \frac{\delta m}{m}\left(-12-\frac{31}{2} \nu\right)(n v)+\mathbf{S} \times \mathbf{v}(-24-19 \nu)+\mathbf{\Sigma} \times \mathbf{v} \frac{\delta m}{m}\left(-12-\frac{19}{2} \nu\right) .
\end{aligned}
$$

Finally, at next-to-next-to-leading order, we get

$$
m \underset{S}{\mathbf{B}_{3.5 \mathrm{PN}}}=\frac{G m}{r^{3}}\left[{ }^{(3.5)} \mathbf{b}_{1}+{ }^{(3.5)} \mathbf{b}_{2} \frac{G m}{r}+{ }^{(3.5)} \mathbf{b}_{3} \frac{G^{2} m^{2}}{r^{2}}\right],
$$

where

$$
\begin{aligned}
& { }^{(3.5)} \mathbf{b}_{1}=(n, \Sigma, v) \mathbf{n}\left[\frac{\delta m}{m}\left(-\frac{105}{4} \nu+\frac{315}{4} \nu^{2}\right)(n v)^{4}+\frac{\delta m}{m}\left(30 \nu-75 \nu^{2}\right)(n v)^{2} v^{2}\right. \\
& \left.+\frac{\delta m}{m}\left(-9 \nu+24 \nu^{2}\right) v^{4}\right] \\
& +(n, S, v) \mathbf{n}\left[\left(-\frac{105}{2} \nu+\frac{315}{2} \nu^{2}\right)(n v)^{4}+\left(60 \nu-150 \nu^{2}\right)(n v)^{2} v^{2}+\left(-18 \nu+48 \nu^{2}\right) v^{4}\right] \\
& +(n, \Sigma, v) \mathbf{v}\left[\frac{\delta m}{m}\left(-\frac{15}{2} \nu-\frac{105}{4} \nu^{2}\right)(n v)^{3}+\frac{\delta m}{m}\left(\frac{3}{8}+\frac{15}{4} \nu+\frac{141}{8} \nu^{2}\right)(n v) v^{2}\right] \\
& +(n, S, v) \mathbf{v}\left[\left(-\frac{15}{2} \nu-\frac{195}{4} \nu^{2}\right)(n v)^{3}+\left(\frac{3}{8}+\frac{27}{8} \nu+\frac{249}{8} \nu^{2}\right)(n v) v^{2}\right] \\
& +\mathbf{n} \times \mathbf{S}\left[\left(\frac{315}{8} \nu-\frac{945}{8} \nu^{2}\right)(n v)^{5}+\left(-\frac{105}{2} \nu+\frac{585}{4} \nu^{2}\right)(n v)^{3} v^{2}\right. \\
& \left.+\left(-\frac{3}{8}+\frac{165}{8} \nu-\frac{441}{8} \nu^{2}\right)(n v) v^{4}\right] \\
& +\mathbf{n} \times \boldsymbol{\Sigma}\left[\frac{\delta m}{m}\left(\frac{105}{4} \nu-\frac{525}{8} \nu^{2}\right)(n v)^{5}+\frac{\delta m}{m}\left(-\frac{75}{2} \nu+\frac{345}{4} \nu^{2}\right)(n v)^{3} v^{2}\right. \\
& \left.+\frac{\delta m}{m}\left(-\frac{3}{8}+\frac{57}{4} \nu-\frac{237}{8} \nu^{2}\right)(n v) v^{4}\right] \\
& +\mathbf{S} \times \mathbf{v}\left[\left(\frac{225}{8} \nu-\frac{585}{8} \nu^{2}\right)(n v)^{4}+\left(-\frac{3}{8}-\frac{255}{8} \nu+\frac{627}{8} \nu^{2}\right)(n v)^{2} v^{2}\right. \\
& \left.+\left(\frac{21}{2} \nu-28 \nu^{2}\right) v^{4}\right] \\
& +\boldsymbol{\Sigma} \times \mathbf{v}\left[\frac{\delta m}{m}\left(15 \nu-\frac{315}{8} \nu^{2}\right)(n v)^{4}+\frac{\delta m}{m}\left(-\frac{3}{8}-\frac{69}{4} \nu+\frac{351}{8} \nu^{2}\right)(n v)^{2} v^{2}\right. \\
& \left.+\frac{\delta m}{m}\left(\frac{11}{2} \nu-14 \nu^{2}\right) v^{4}\right]
\end{aligned}
$$




$$
\begin{aligned}
& { }^{(3.5)} \mathbf{b}_{2}=(n, \Sigma, v) \mathbf{n}\left[\frac{\delta m}{m}\left(\frac{3147}{8} \nu+\frac{255}{4} \nu^{2}\right)(n v)^{2}+\frac{\delta m}{m}\left(-\frac{131}{8} \nu-19 \nu^{2}\right) v^{2}\right] \\
& +(n, S, v) \mathbf{n}\left[\left(\frac{1635}{2} \nu+117 \nu^{2}\right)(n v)^{2}+\left(-\frac{217}{4} \nu-28 \nu^{2}\right) v^{2}\right] \\
& +(n, \Sigma, v) \mathbf{v} \frac{\delta m}{m}\left(-\frac{381}{2} \nu-25 \nu^{2}\right)(n v)+(n, S, v) \mathbf{v}\left(-\frac{777}{2} \nu-\frac{87}{2} \nu^{2}\right)(n v) \\
& +\mathbf{n} \times \mathbf{S}\left[\left(-\frac{1215}{2} \nu-105 \nu^{2}\right)(n v)^{3}+\left(\frac{1067}{4} \nu+\frac{79}{2} \nu^{2}\right)(n v) v^{2}\right] \\
& +\mathbf{n} \times \boldsymbol{\Sigma}\left[\frac{\delta m}{m}\left(-\frac{2193}{8} \nu-\frac{279}{4} \nu^{2}\right)(n v)^{3}+\frac{\delta m}{m}\left(\frac{945}{8} \nu+23 \nu^{2}\right)(n v) v^{2}\right] \\
& +\mathbf{S} \times \mathbf{v}\left[\left(-352 \nu-\frac{123}{2} \nu^{2}\right)(n v)^{2}+\left(\frac{197}{4} \nu+14 \nu^{2}\right) v^{2}\right] \\
& +\Sigma \times \mathbf{v}\left[\frac{\delta m}{m}\left(-\frac{1325}{8} \nu-\frac{147}{4} \nu^{2}\right)(n v)^{2}+\frac{\delta m}{m}\left(\frac{177}{8} \nu+7 \nu^{2}\right) v^{2}\right], \\
& { }^{(3.5)} \mathbf{b}_{3}=(n, \Sigma, v) \mathbf{n} \frac{\delta m}{m}\left(-\frac{111}{2}-\frac{441}{4} \nu+5 \nu^{2}\right)+(n, S, v) \mathbf{n}\left(-\frac{195}{2}-\frac{749}{4} \nu+8 \nu^{2}\right) \\
& +\mathbf{n} \times \mathbf{S}\left(\frac{121}{2}+65 \nu-8 \nu^{2}\right)(n v)+\mathbf{n} \times \mathbf{\Sigma} \frac{\delta m}{m}\left(\frac{57}{2}+\frac{85}{4} \nu-6 \nu^{2}\right)(n v) \\
& +\mathbf{S} \times \mathbf{v}\left(\frac{105}{2}+\frac{137}{2} \nu\right)+\mathbf{\Sigma} \times \mathbf{v} \frac{\delta m}{m}\left(\frac{57}{2}+\frac{65}{2} \nu\right) \text {. }
\end{aligned}
$$

\section{Conserved integrals of the motion}

As already verified in Paper I, the dynamics associated with the next-to-next-to-leading spin orbit terms at 3.5PN order is purely conservative (i.e. there exists a conserved energy at that order) and, in the harmonic coordinate system we are using, is manifestly invariant under Poincaré transformations. Therefore, it admits the usual set of Noetherian integrals of motion associated with the Poincaré group (energy, linear momentum, angular momentum, center-of-mass integral).

These integrals can be computed in a general frame from the acceleration given in Eqs. (6.7)-(6.12) of Paper I (translated to our conserved-norm spin variable) on the one hand, and the 3PN precession equation given previously in Section IIB on the other hand, by using the method of undetermined coefficients as was done for the energy in Paper I. Note that the precession equation is needed at 3PN order only for the total angular momentum while the $2 \mathrm{PN}$ order is sufficient for the other quantities.

However, a technical difficulty of the method of undetermined coefficients is that it must deal with the fact that a given expression does not admit a unique writing due to the existence of dimensional identities relating the different vectors of the problem. For this reason, we have chosen here a more direct approach consisting in translating the expressions obtained in Ref. [40] from a reduced Hamiltonian formalism in ADM-type coordinates and then verifying that they are indeed conserved as a consequence of our equations of motion. 
Note that in the Hamiltonian formalism, the total linear momentum and the total angular momentum are trivially expressed in terms of the canonical variables. The translation to our variables is performed by applying to the ADM results the contact transformation derived at the required order in Section VII D of Paper I. For the angular momentum, we also need the relation between our spin variables and the canonical spin, 1PN order beyond that given in Paper I. The derivation of this $3 \mathrm{PN}$ correspondence between both sets of spin variables is presented in Appendix A.

The resulting expressions in a general frame happen to be very long, hence we directly display them reduced to the CM frame, in which, by definition, only the energy and the angular momentum do not vanish. The latter quantity being still relatively lengthy, it is relegated to Appendix D. The energy in the CM frame takes the form

$$
\begin{gathered}
E=\nu\left\{e_{\mathrm{N}}+\frac{1}{c^{2}} e_{1 \mathrm{PN}}+\frac{1}{c^{3}} \underset{S}{e_{1.5 \mathrm{PN}}}+\frac{1}{c^{4}}\left[e_{2 \mathrm{PN}}+\underset{\left.S S^{2 \mathrm{PN}}\right]+\frac{1}{c^{5}}{\underset{S}{2.5 \mathrm{PN}}}_{2}}{\left.+\frac{1}{c^{6}}\left[e_{3 \mathrm{PN}}+\underset{S S}{e} 3 \mathrm{PN}\right]+\frac{1}{c^{7}} e_{S} 3.5 \mathrm{PN}+\mathcal{O}\left(\frac{1}{c^{8}}\right)\right\},}\right.\right.
\end{gathered}
$$

where the non-spin terms can be found for instance in Ref. [11]. For the spin-orbit contributions, we get at leading order

$$
{\underset{S}{1.5 \mathrm{PN}}}_{1}=\frac{G m}{r^{2}}\left\{-\frac{\delta m}{m}(n, \Sigma, v)-(n, S, v)\right\},
$$

which agrees with the standard results [23, 28], and at next-to leading order

$$
\begin{aligned}
\underset{S}{e_{2.5 \mathrm{PN}}=} & \frac{G m}{r^{2}}\left\{(n, \Sigma, v) \frac{\delta m}{m}\left(-\frac{1}{2}+\frac{5}{2} \nu\right) v^{2}+(n, S, v)\left[\frac{3}{2} \nu(n v)^{2}+\left(\frac{3}{2}+\frac{3}{2} \nu\right) v^{2}\right]\right\} \\
& +\frac{G^{2} m^{2}}{r^{3}} \nu\left\{-\frac{3}{2} \frac{\delta m}{m}(n, \Sigma, v)-2(n, S, v)\right\}
\end{aligned}
$$

which differs from [23, 28] due to the deviation in the spin variables beyond leading order. For the next-to-next-to leading order, we arrive at

$$
\begin{aligned}
{\underset{S}{S}}_{3.5 \mathrm{PN}}=\frac{G m}{r^{2}}\{ & (n, \Sigma, v)\left[\frac{\delta m}{m}\left(-\frac{3}{8}+\frac{53}{8} \nu-\frac{99}{8} \nu^{2}\right) v^{4}+\frac{\delta m}{m}\left(-3 \nu-\frac{3}{2} \nu^{2}\right)(n v)^{2} v^{2}\right. \\
& \left.+\frac{15}{8} \nu^{2} \frac{\delta m}{m}(n v)^{4}\right]+(n, S, v)\left[\left(-\frac{15}{8} \nu+\frac{45}{8} \nu^{2}\right)(n v)^{4}\right. \\
& \left.\left.+\left(\frac{3}{4} \nu-\frac{51}{4} \nu^{2}\right)(n v)^{2} v^{2}+\left(\frac{21}{8}-\frac{31}{8} \nu-\frac{55}{8} \nu^{2}\right) v^{4}\right]\right\} \\
+ & \frac{G^{2} m^{2}}{r^{3}}\left\{(n, \Sigma, v)\left[\frac{\delta m}{m}\left(\frac{179}{8} \nu+\frac{3}{2} \nu^{2}\right)(n v)^{2}+\frac{\delta m}{m}\left(-2-\frac{145}{8} \nu+\frac{41}{4} \nu^{2}\right) v^{2}\right]\right. \\
& \left.+(n, S, v)\left[\left(\frac{187}{4} \nu+5 \nu^{2}\right)(n v)^{2}+\left(6-\frac{143}{4} \nu+10 \nu^{2}\right) v^{2}\right]\right\} \\
+ & \frac{G^{3} m^{3}}{r^{4}}\left\{(n, \Sigma, v) \frac{\delta m}{m}\left(-\frac{1}{2}-\frac{17}{2} \nu-\frac{3}{2} \nu^{2}\right)+(n, S, v)\left(-\frac{1}{2}-\frac{79}{4} \nu-2 \nu^{2}\right)\right\} .
\end{aligned}
$$




\section{E. Near-zone metric at the location of a particle}

The post-Newtonian near-zone metric up to order $3.5 \mathrm{PN}$ is parametrized in terms of the elementary potentials according to Eqs. (2.16). The next-to-next-to-leading spin-orbit effects correspond to terms of order $\mathcal{O}\left(1 / c^{9}\right)$ in $g_{00}, \mathcal{O}\left(1 / c^{8}\right)$ in $g_{0 i}$ and $\mathcal{O}\left(1 / c^{7}\right)$ in $g_{i j}$. However, because of the complicated non-linear structure of the source terms, analytical solutions to the appropriate order can only be obtained for some of these potentials using the technique described in details in Sections IIIC and D of Paper I. As a result, the spin-orbit contributions to the near-zone metric evaluated at an arbitrary field point in the near zone is computable in analytic closed form only up to $\mathcal{O}\left(1 / c^{7}\right)$ for $g_{00}, \mathcal{O}\left(1 / c^{6}\right)$ for $g_{0 i}$ and $\mathcal{O}\left(1 / c^{7}\right)$ for $g_{i j}$. The corresponding expressions will be given in Section IIIF By contrast, the value of all these potentials at the location of each of the two point particles, defined by means of the pure Hadamard-Schwartz regularization procedure, has been calculated explicitly by using the tools presented in Section IIID of Paper I. This has enabled us to obtain an expression for the regularized metric at the particle positions to the desired orders. In addition to the regularized potentials already obtained in Paper I, we shall now need the spin parts of $(\hat{X})_{1}$ to next-to-leading order, as well as $(\hat{T})_{1}$ to leading order, which we evaluate in a similar fashion. We define

$$
\begin{aligned}
& \left(g_{S}^{g} 00\right)_{1}=\frac{1}{c^{5}}\left(\underset{S}{g_{00}^{1.5 \mathrm{PN}}}\right)_{1}+\frac{1}{c^{7}}\left(\underset{S}{g_{00}^{2.5 \mathrm{PN}}}\right)_{1}+\frac{1}{c^{8}}\left(\underset{S}{g_{00}^{3 \mathrm{PN}}}\right)_{1}+\frac{1}{c^{9}}\left(\underset{S}{g_{00}^{3.5 \mathrm{PN}}}\right)_{1}+\mathcal{O}\left(\frac{1}{c^{10}}\right), \\
& \left(g_{S} 0 i\right)_{1}=\frac{1}{c^{4}}\left({\underset{S}{0}}_{0 i}^{1.5 \mathrm{PN}}\right)_{1}+\frac{1}{c^{6}}\left({\underset{S}{0 i}}_{0 i}^{2.5 \mathrm{PN}}\right)_{1}+\frac{1}{c^{7}}\left(\underset{S}{g_{0 i}^{3 \mathrm{PN}}}\right)_{1}+\frac{1}{c^{8}}\left(\underset{S}{g_{0 i}^{3.5 \mathrm{PN}}}\right)_{1}+\mathcal{O}\left(\frac{1}{c^{9}}\right), \\
& \left(g_{S} i j\right)_{1}=\frac{1}{c^{5}}\left(g_{S}^{2.5 \mathrm{PNN}}\right)_{1}+\frac{1}{c^{7}}\left(g_{S}^{3.5 \mathrm{PN}}\right)_{1}+\mathcal{O}\left(\frac{1}{c^{8}}\right)
\end{aligned}
$$

The non-spin parts can be found in Ref. [50]. The spin-orbit contributions at 3PN order, namely the terms $\sim 1 / c^{8}$ in ${ }_{S} g_{00}$ and $\sim 1 / c^{7}$ in ${ }_{S} g_{0 i}$, are actually pure coordinate effects. These terms can be eliminated by means of a gauge transformation as verified in Appendix E (extending Ref. [51]). After reduction to the center-of-mass frame, we are led to the following spin parts.

\section{Metric component $g_{00}$}

$$
\begin{aligned}
& \left(g_{S}^{1.5 \mathrm{PN}}\right)_{1}=\frac{G \nu}{r^{2}}\left\{(n, \Sigma, v)\left(2+2 \frac{\delta m}{m}\right)+4(n, S, v)\right\}, \\
& \left(g_{S}^{2.5 \mathrm{PN}}\right)_{1}=\frac{G \nu}{r^{2}}\left\{(n, S, v)\left[\left(-3+6 \nu-3 \frac{\delta m}{m}\right)(n v)^{2}+\left(3-8 \nu+\frac{\delta m}{m}\right) v^{2}\right]\right. \\
& +(n, \Sigma, v)\left[\left(-3+9 \nu+\frac{\delta m}{m}(-3+3 \nu)\right)(n v)^{2}\right. \\
& \left.\left.+\left(2-6 \nu+\frac{\delta m}{m}(2-4 \nu)\right) v^{2}\right]\right\}
\end{aligned}
$$




$$
\begin{aligned}
& +\frac{G^{2} m \nu}{r^{3}}\left\{(n, \Sigma, v)\left(-\frac{5}{2}+8 \nu+\frac{\delta m}{m}\left(-\frac{17}{2}+2 \nu\right)\right)\right. \\
& \left.+(n, S, v)\left(-14+4 \nu+\frac{\delta m}{m}\right)\right\} \text {, } \\
& \left(\begin{array}{l}
g_{S}^{3 \mathrm{PN}} \\
00
\end{array}\right)_{1}=\frac{G^{2} m \nu}{r^{3}}\left\{(n, \Sigma, v)\left(2-10 \frac{\delta m}{m}\right)(n v)-24(n v)(n, S, v)\right\}, \\
& \left(\begin{array}{c}
g_{S}^{3.5 \mathrm{PN}} \\
00
\end{array}\right)_{1}=\frac{G \nu}{r^{2}}\left\{( n , \Sigma , v ) \left[\left(\frac{15}{4}-\frac{75}{4} \nu+\frac{75}{4} \nu^{2}+\frac{\delta m}{m}\left(\frac{15}{4}-\frac{45}{4} \nu+\frac{15}{4} \nu^{2}\right)\right)(n v)^{4}\right.\right. \\
& +\left(-6+\frac{69}{2} \nu-48 \nu^{2}+\frac{\delta m}{m}\left(-6+\frac{45}{2} \nu-15 \nu^{2}\right)\right)(n v)^{2} v^{2} \\
& \left.+\left(2-13 \nu+22 \nu^{2}+\frac{\delta m}{m}\left(2-\frac{21}{2} \nu+14 \nu^{2}\right)\right) v^{4}\right] \\
& +(n, S, v)\left[\left(\frac{15}{4}-15 \nu+\frac{15}{2} \nu^{2}+\frac{\delta m}{m}\left(\frac{15}{4}-\frac{15}{2} \nu\right)\right)(n v)^{4}\right. \\
& +\left(-6+\frac{57}{2} \nu-30 \nu^{2}+\frac{\delta m}{m}\left(-6+\frac{33}{2} \nu\right)\right)(n v)^{2} v^{2} \\
& \left.\left.+\left(\frac{11}{4}-17 \nu+28 \nu^{2}+\frac{\delta m}{m}\left(\frac{5}{4}-4 \nu\right)\right) v^{4}\right]\right\} \\
& +\frac{G^{2} m \nu}{r^{3}}\left\{( n , \Sigma , v ) \left[\left(\frac{43}{8}+\frac{39}{2} \nu-60 \nu^{2}+\frac{\delta m}{m}\left(\frac{119}{8}+24 \nu-12 \nu^{2}\right)\right) v^{2}\right.\right. \\
& \left.+\left(-\frac{145}{8}-11 \nu+30 \nu^{2}+\frac{\delta m}{m}\left(-\frac{645}{8}-\frac{41}{2} \nu+8 \nu^{2}\right)\right)(n v)^{2}\right] \\
& +(n, S, v)\left[\left(-\frac{269}{2}-20 \nu+16 \nu^{2}+\frac{\delta m}{m}\left(-\frac{19}{4}-\nu\right)\right)(n v)^{2}\right. \\
& \left.\left.+\left(\frac{59}{2}+28 \nu-24 \nu^{2}+\frac{\delta m}{m}\left(\frac{25}{4}+4 \nu\right)\right) v^{2}\right]\right\} \\
& +\frac{G^{3} m^{2} \nu}{r^{4}}\left\{(n, \Sigma, v)\left(-\frac{17}{2}-\frac{33}{10} \nu+32 \nu^{2}+\frac{\delta m}{m}\left(-14-\frac{31}{2} \nu+2 \nu^{2}\right)\right)\right. \\
& \left.+(n, S, v)\left(-35-30 \nu+4 \nu^{2}+\frac{\delta m}{m}\left(-\frac{7}{2}-7 \nu\right)\right)\right\} \text {. }
\end{aligned}
$$

2. Metric components $g_{0 i}$

$$
\begin{aligned}
& \left(\underset{S}{g_{0 i}^{1.5 \mathrm{PN}}}\right)_{1}=\frac{G}{r^{2}}\left\{\varepsilon^{i j k} n^{j} S^{k}\left(1-\frac{\delta m}{m}\right)+2 \nu \varepsilon^{i j k} n^{j} \Sigma^{k}\right\}, \\
& \left(g_{S}^{2.5 \mathrm{PN}}\right)_{1}=\frac{G}{r^{2}} \nu\left\{(n, \Sigma, v) v^{i}\left(\frac{3}{2}-3 \nu+\frac{3}{2} \frac{\delta m}{m}\right)+(n, S, v) v^{i}\left(\frac{3}{2}+\frac{3}{2} \frac{\delta m}{m}\right)\right. \\
& +\varepsilon^{i j k} n^{j} S^{k}\left[\left(-\frac{3}{2}-\frac{3}{2} \frac{\delta m}{m}\right)(n v)^{2}+\left(\frac{1}{2}+\frac{1}{2} \frac{\delta m}{m}\right) v^{2}\right]
\end{aligned}
$$




$$
\begin{aligned}
& +\varepsilon^{i j k} n^{j} \Sigma^{k}\left[\left(-\frac{3}{2}+3 \nu-\frac{3}{2} \frac{\delta m}{m}\right)(n v)^{2}+\left(\frac{1}{2}-\nu+\frac{1}{2} \frac{\delta m}{m}\right) v^{2}\right] \\
& \left.+\varepsilon^{i j k} S^{j} v^{k}\left(-\frac{1}{2}-\frac{1}{2} \frac{\delta m}{m}\right)(n v)+\varepsilon^{i j k} \Sigma^{j} v^{k}\left(-\frac{1}{2}+\nu-\frac{1}{2} \frac{\delta m}{m}\right)(n v)\right\} \\
& +\frac{G^{2} m}{r^{3}}\left\{\varepsilon^{i j k} n^{j} S^{k}\left(-1+\frac{\delta m}{m}\right)+\varepsilon^{i j k} n^{j} \Sigma^{k}\left(-2 \nu-2 \nu \frac{\delta m}{m}\right)\right\}, \\
& \left(g_{S}^{g_{0 i}^{3 \mathrm{PN}}}\right)_{1}=\frac{G^{2} m}{r^{3}} \nu\left\{2(n v) \varepsilon^{i j k} n^{j} \Sigma^{k}+\frac{2}{3} \varepsilon^{i j k} \Sigma^{j} v^{k}\right\}, \\
& \left(g_{S}^{g_{0 i}^{3.5 \mathrm{PN}}}\right)_{1}=\frac{G}{r^{2}} \nu\left\{( n , \Sigma , v ) v ^ { i } \left[\left(-\frac{9}{4}+9 \nu-\frac{9}{2} \nu^{2}+\frac{\delta m}{m}\left(-\frac{9}{4}+\frac{9}{2} \nu\right)\right)(n v)^{2}\right.\right. \\
& \left.+\left(\frac{13}{8}-8 \nu+\frac{37}{4} \nu^{2}+\frac{\delta m}{m}\left(\frac{13}{8}-\frac{19}{4} \nu\right)\right) v^{2}\right] \\
& +(n, S, v) v^{i}\left[\left(-\frac{9}{4}+\frac{27}{4} \nu+\frac{\delta m}{m}\left(-\frac{9}{4}+\frac{9}{4} \nu\right)\right)(n v)^{2}\right. \\
& \left.+\left(\frac{13}{8}-\frac{39}{8} \nu+\frac{\delta m}{m}\left(\frac{13}{8}-\frac{37}{8} \nu\right)\right) v^{2}\right] \\
& +\varepsilon^{i j k} n^{j} S^{k}\left[\left(\frac{15}{8}-\frac{45}{8} \nu+\frac{\delta m}{m}\left(\frac{15}{8}-\frac{15}{8} \nu\right)\right)(n v)^{4}\right. \\
& +\left(-\frac{9}{4}+\frac{27}{4} \nu+\frac{\delta m}{m}\left(-\frac{9}{4}+\frac{21}{4} \nu\right)\right)(n v)^{2} v^{2} \\
& \left.+\left(\frac{3}{8}-\frac{9}{8} \nu+\frac{\delta m}{m}\left(\frac{3}{8}-\frac{11}{8} \nu\right)\right) v^{4}\right] \\
& +\varepsilon^{i j k} n^{j} \Sigma^{k}\left[\left(\frac{15}{8}-\frac{15}{2} \nu+\frac{15}{4} \nu^{2}+\frac{\delta m}{m}\left(\frac{15}{8}-\frac{15}{4} \nu\right)\right)(n v)^{4}\right. \\
& +\left(-\frac{9}{4}+\frac{21}{2} \nu-\frac{21}{2} \nu^{2}+\frac{\delta m}{m}\left(-\frac{9}{4}+6 \nu\right)\right)(n v)^{2} v^{2} \\
& \left.+\left(\frac{3}{8}-2 \nu+\frac{11}{4} \nu^{2}+\frac{\delta m}{m}\left(\frac{3}{8}-\frac{5}{4} \nu\right)\right) v^{4}\right] \\
& +\varepsilon^{i j k} S^{j} v^{k}\left[\left(\frac{3}{4}-\frac{9}{4} \nu+\frac{\delta m}{m}\left(\frac{3}{4}-\frac{3}{4} \nu\right)\right)(n v)^{3}\right. \\
& \left.+\left(-\frac{5}{8}+\frac{15}{8} \nu+\frac{\delta m}{m}\left(-\frac{5}{8}+\frac{13}{8} \nu\right)\right)(n v) v^{2}\right] \\
& +\varepsilon^{i j k} \Sigma^{j} v^{k}\left[\left(\frac{3}{4}-3 \nu+\frac{3}{2} \nu^{2}+\frac{\delta m}{m}\left(\frac{3}{4}-\frac{3}{2} \nu\right)\right)(n v)^{3}\right. \\
& \left.\left.+\left(-\frac{5}{8}+3 \nu-\frac{13}{4} \nu^{2}+\frac{\delta m}{m}\left(-\frac{5}{8}+\frac{7}{4} \nu\right)\right)(n v) v^{2}\right]\right\} \\
& +\frac{G^{2} m}{r^{3}} \nu\left\{(n, \Sigma, v) n^{i}\left(-\frac{59}{2}+\frac{363}{4} \nu-3 \nu^{2}+\frac{\delta m}{m}\left(\frac{37}{2}+\frac{103}{4} \nu\right)\right)(n v)\right. \\
& +(n, S, v) n^{i}\left(\frac{91}{2}+50 \nu+\frac{\delta m}{m}\left(-\frac{89}{2}+\frac{3}{2} \nu\right)\right)(n v)
\end{aligned}
$$




$$
\begin{aligned}
& +(n, \Sigma, v) v^{i}\left(3+\frac{15}{2} \nu-6 \nu^{2}+\frac{\delta m}{m}\left(-8+\frac{21}{2} \nu\right)\right) \\
& +(n, S, v) v^{i}\left(-12+4 \nu+\frac{\delta m}{m}(-1+3 \nu)\right) \\
& +\varepsilon^{i j k} n^{j} S^{k}\left[\left(-\frac{131}{2}-36 \nu+\frac{\delta m}{m}\left(\frac{79}{2}-\frac{9}{2} \nu\right)\right)(n v)^{2}\right. \\
& \left.+\left(\frac{35}{2}+\frac{\delta m}{m}(-5+\nu)\right) v^{2}\right] \\
& +\varepsilon^{i j k} n^{j} \Sigma^{k}\left[\left(11-\frac{333}{4} \nu+9 \nu^{2}+\frac{\delta m}{m}\left(-29-\frac{97}{4} \nu\right)\right)(n v)^{2}\right. \\
& \left.+\left(-\frac{5}{4}+\frac{25}{2} \nu-2 \nu^{2}+\frac{\delta m}{m}\left(\frac{27}{4}+\frac{7}{2} \nu\right)\right) v^{2}\right] \\
& +\varepsilon^{i j k} S^{j} v^{k}\left(-32-28 \nu+\frac{\delta m}{m}\left(\frac{53}{2}-\frac{3}{2} \nu\right)\right)(n v) \\
& \left.+\varepsilon^{i j k} \Sigma^{j} v^{k}\left(\frac{43}{4}-\frac{215}{4} \nu+3 \nu^{2}+\frac{\delta m}{m}\left(-\frac{57}{4}-\frac{63}{4} \nu\right)\right)(n v)\right\} \\
& +\frac{G^{3} m^{2}}{r^{4}}\left\{\varepsilon^{i j k} n^{j} S^{k}\left(1+\frac{5}{4} \nu-8 \nu^{2}+\frac{\delta m}{m}\left(-1-\frac{11}{4} \nu\right)\right)\right. \\
& \left.+\varepsilon^{i j k} n^{j} \Sigma^{k}\left(\frac{89}{20} \nu+\frac{13}{2} \nu^{2}+\frac{\delta m}{m}\left(-\frac{11}{20} \nu-6 \nu^{2}\right)\right)\right\} .
\end{aligned}
$$

3. Metric components $g_{i j}$

$$
\begin{aligned}
& \left(g_{S}^{2.5 \mathrm{PN}}\right)_{1}=\frac{G \nu}{r^{2}}\left\{v^{(i} \varepsilon^{j) k l} n^{k} \Sigma^{l}\left(2+2 \frac{\delta m}{m}\right)+4 v^{(i} \varepsilon^{j) k l} n^{k} S^{l}\right\} \\
& \left(g_{S}^{3.5 \mathrm{PN}}\right)_{1}=\frac{G \nu}{r^{2}}\left\{(n, \Sigma, v) v^{i} v^{j}\left(1-3 \nu+\frac{\delta m}{m}(1-\nu)\right)+(n, S, v) v^{i} v^{j}\left(1-2 \nu+\frac{\delta m}{m}\right)\right. \\
& +v^{(i} \varepsilon^{j) k l} n^{k} \Sigma^{l}\left[\left(-3+9 \nu+\frac{\delta m}{m}(-3+3 \nu)\right)(n v)^{2}\right. \\
& \left.+\left(1-3 \nu+\frac{\delta m}{m}(1-3 \nu)\right) v^{2}\right]+v^{(i} \varepsilon^{j) k l} S^{k} v^{l}\left(-1+2 \nu-\frac{\delta m}{m}\right)(n v) \\
& +v^{(i} \varepsilon^{j) k l} n^{k} S^{l}\left[\left(-3+6 \nu-3 \frac{\delta m}{m}\right)(n v)^{2}+(2-6 \nu) v^{2}\right] \\
& \left.+v^{(i} \varepsilon^{j) k l} \Sigma^{k} v^{l}\left(-1+3 \nu+\frac{\delta m}{m}(-1+\nu)\right)(n v)\right\} \\
& +\frac{G^{2} m \nu}{r^{3}}\left\{(n, \Sigma, v) \delta^{i j}\left(\frac{5}{2}+\frac{17}{2} \frac{\delta m}{m}\right)+(n, \Sigma, v) n^{i} n^{j}\left(-8-56 \frac{\delta m}{m}\right)\right. \\
& +17(n, S, v) \delta^{i j}+n^{(i} \varepsilon^{j) k l} n^{k} \Sigma^{l}\left(3+4 \nu+\frac{\delta m}{m}(51+2 \nu)\right)(n v)
\end{aligned}
$$




$$
\begin{aligned}
& -112(n, S, v) n^{i} n^{j}+n^{(i} \varepsilon^{j) k l} n^{k} S^{l}\left(103+4 \nu-\frac{\delta m}{m}\right)(n v) \\
& +n^{(i} \varepsilon^{j) k l} \Sigma^{k} v^{l}\left(6+34 \frac{\delta m}{m}\right)+v^{(i} \varepsilon^{j) k l} n^{k} S^{l}\left(-23+4 \nu+\frac{\delta m}{m}\right) \\
& \left.+68 n^{(i} \varepsilon^{j) k l} S^{k} v^{l}+v^{(i} \varepsilon^{j) k l} n^{k} \Sigma^{l}\left(-1+8 \nu+\frac{\delta m}{m}(-13+2 \nu)\right)\right\} .
\end{aligned}
$$

\section{F. Near-zone metric in the bulk}

Let us now turn to the spin part of the near-zone metric up to orders $\mathcal{O}\left(1 / c^{7}\right)$ for $g_{00}$, $\mathcal{O}\left(1 / c^{6}\right)$ for $g_{0 i}$ and $\mathcal{O}\left(1 / c^{7}\right)$ for $g_{i j}$. As mentioned above, the wave equations defining the potentials at play up to these orders can be analytically integrated. We refer to Section $\mathrm{V}$ of [50] for a thorough presentation of the relevant method. The non-spin parts of the metric up to the same orders can be found in Ref. [50].

The metric at a given arbitrary field point $\mathbf{x}$ is most conveniently expressed using the distances to the two particles $r_{1}=\left|\mathbf{x}-\mathbf{y}_{\mathbf{1}}\right|$ and $r_{2}=\left|\mathbf{x}-\mathbf{y}_{\mathbf{2}}\right|$, as well as the unit vectors $\mathbf{n}_{1}=\left(\mathbf{x}-\mathbf{y}_{\mathbf{1}}\right) / r_{1}$ and $\mathbf{n}_{2}=\left(\mathbf{x}-\mathbf{y}_{\mathbf{2}}\right) / r_{2}$ pointing from the particles to the field point. In order to avoid any confusion, let us recall that, as in the rest of the present Section III, $r \equiv r_{12}$ and $\mathbf{n} \equiv n_{12}$ respectively denote the radial separation between the two bodies and the unit vector pointing from body 2 to body 1 .

One key ingredient in the computation of the relevant potentials is the function $g \equiv$ $\ln \left(r_{1}+r_{2}+r\right)$, which satisfies $\Delta g=1 /\left(r_{1} r_{2}\right)$, where it is understood that the Laplacian operator acts on functions of $\mathbf{x}$. In the spin part of the metric presented below, $g$ appears within terms of the form $\left(\partial_{1 i j} \partial_{2 k} g\right)$, where $\partial_{1 i}$ and $\partial_{2 i}$ denote the partial derivatives with respect to $y_{1}^{i}$ and $y_{2}^{i}$. An explicit form for such terms is straightforwardly obtained by expanding these derivatives but we shall keep them factorized in an effort to reduce the length of our expressions. Finally, we find that using the spin variables $\mathbf{S}_{1}$ and $\mathbf{S}_{\mathbf{2}}$ instead of their combinations $\boldsymbol{\Sigma}$ and $\mathbf{S}$ leads to much more compact expressions and, since the metric has to be symmetric under the exchange $1 \leftrightarrow 2$, we can then further shorten our formulae by only explicitly giving the contribution containing $\mathbf{S}_{1}$. Note that in practice, the operation $1 \leftrightarrow 2$ implies the replacements $\delta m \rightarrow-\delta m, \mathbf{v} \rightarrow-\mathbf{v}$ and $\mathbf{n} \rightarrow-\mathbf{n}$ in addition to those where the indices 1 and 2 explicitly appear.

1. Metric component 00

$$
\begin{aligned}
g_{S} 00= & \frac{1}{c^{5}} \frac{G}{r_{1}^{2}}\left(n_{1}, S_{1}, v\right)\left(-2+2 \frac{\delta m}{m}\right)+\frac{1}{c^{7}}[ \\
& \frac{G}{r_{1}^{2}}\left(n_{1}, S_{1}, v\right)\left[\left(-2+6 \nu+\frac{\delta m}{m}(2-4 \nu)\right) v^{2}+\left(3-9 \nu+\frac{\delta m}{m}(-3+3 \nu)\right)\left(n_{1} v\right)^{2}\right] \\
& +\frac{G^{2} m}{r_{1}^{2} r_{2}}\left(n_{1}, S_{1}, v\right)\left(4-4 \frac{\delta m}{m}\right)+\frac{G^{2} m}{r_{1}^{3}} 4 \nu\left(n_{1}, S_{1}, v\right)
\end{aligned}
$$




$$
\begin{aligned}
& +\frac{G^{2} m}{r r_{1}^{2}} \nu\left\{\left(n_{1}, S_{1}, v\right)\left(-8+2 \frac{\delta m}{m}\right)-2 \frac{\delta m}{m}(n v)\left(n_{1}, n, S_{1}\right)\right\} \\
& +\frac{G^{2} m}{r^{2} r_{2}}\left\{\left(n, S_{1}, v\right)\left(2-4 \nu+\frac{\delta m}{m}(-2+4 \nu)\right)+\left(n_{2}, n, S_{1}\right)\left(\nu-\nu \frac{\delta m}{m}\right)\left(n_{2} v\right)\right\} \\
& +\frac{G^{2} m}{r^{2} r_{1}}\left\{\left(n, S_{1}, v\right)\left(-3+2 \nu+\frac{\delta m}{m}(3-4 \nu)\right)+\left(n_{1}, S_{1}, v\right)\left(-1+2 \nu+\frac{\delta m}{m}\right)\left(n_{1} n\right)\right. \\
& \left.+\left(n_{1}, n, S_{1}\right)\left(2-5 \nu+\frac{\delta m}{m}(-2+\nu)\right)\left(n_{1} v\right)\right\} \\
& +\frac{G^{2} m}{r^{3}}\left\{\left(n, S_{1}, v\right)\left[\left(3-3 \frac{\delta m}{m}\right)\left(n_{1} n\right)+\left(-3+3 \frac{\delta m}{m}\right)\left(n_{2} n\right)\right]\right. \\
& +\left(n_{1}, S_{1}, v\right)\left(-\frac{5}{2}+\frac{5}{2} \frac{\delta m}{m}\right)+\left(n_{2}, S_{1}, v\right)\left(2-2 \frac{\delta m}{m}\right) \\
& \left.+\left(n_{1}, n, S_{1}\right)\left(-\frac{9}{2}+\frac{9}{2} \frac{\delta m}{m}\right)(n v)+\left(n_{2}, n, S_{1}\right)\left(3-3 \frac{\delta m}{m}\right)(n v)\right\} \\
& +G^{2} m\left\{16 \nu\left(\underset{1}{\partial_{j a}}{\underset{2}{\partial}}_{b} g\right) \varepsilon^{i a b} S_{1}^{i} v^{j}+\left(\underset{1}{\partial_{b}} \underset{2}{\partial_{j a} g}\right) \varepsilon^{i a b} S_{1}^{i} v^{j}\left(-4+4 \frac{\delta m}{m}\right)\right.
\end{aligned}
$$

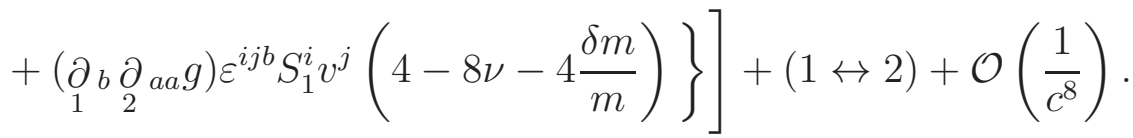

2. Metric components $0 i$

$$
\begin{aligned}
\underset{S}{g_{0 i}=} & \frac{2}{c^{4}} \frac{G}{r_{1}^{2}} \varepsilon^{i j k} n_{1}^{j} S_{1}^{k}+\frac{1}{c^{6}}[ \\
& \frac{G}{r_{1}^{2}}\left\{\left(n_{1}, S_{1}, v\right) v^{i}\left(\frac{3}{2}-3 \nu-\frac{3}{2} \frac{\delta m}{m}\right)+\varepsilon^{i j k} S_{1}^{j} v^{k}\left(-\frac{1}{2}+\nu+\frac{1}{2} \frac{\delta m}{m}\right)\left(n_{1} v\right)\right. \\
& \left.+\varepsilon^{i j k} n_{1}^{j} S_{1}^{k}\left[\left(\frac{1}{2}-\nu-\frac{1}{2} \frac{\delta m}{m}\right) v^{2}+\left(-\frac{3}{2}+3 \nu+\frac{3}{2} \frac{\delta m}{m}\right)\left(n_{1} v\right)^{2}\right]\right\} \\
& +\frac{G^{2} m}{r_{1}^{3}} \varepsilon^{i j k} n_{1}^{j} S_{1}^{k}\left(-1-\frac{\delta m}{m}\right)+\frac{G^{2} m}{r r_{1}^{2}} \varepsilon^{i j k} n_{1}^{j} S_{1}^{k}\left(-2+2 \frac{\delta m}{m}\right)+\frac{G^{2} m}{r^{2} r_{2}} 4 \nu \varepsilon^{i j k} n^{j} S_{1}^{k} \\
& +\frac{G^{2} m}{r^{2} r_{1}}\left\{\varepsilon^{i j k} n^{j} S_{1}^{k}\left(-\frac{1}{2}-4 \nu+\frac{1}{2} \frac{\delta m}{m}\right)+\varepsilon^{i j k} n_{1}^{j} S_{1}^{k}\left(\frac{1}{2}-\frac{1}{2} \frac{\delta m}{m}\right)\left(n_{1} n\right)\right\} \\
& \left.+4 G^{2} m\left(\underset{1}{\partial_{i a}}{ }_{2}{ }_{b} g\right) \varepsilon^{j a b} S_{1}^{j}\left(1-\frac{\delta m}{m}\right)\right]+(1 \leftrightarrow 2)+\mathcal{O}\left(\frac{1}{c^{7}}\right) .
\end{aligned}
$$


3. Metric components ij

$$
\begin{aligned}
& g_{S} i j=\frac{1}{c^{5}} \frac{G}{r_{1}^{2}} v^{(i} \varepsilon^{j) k l} n_{1}^{k} S_{1}^{l}\left(-2+2 \frac{\delta m}{m}\right)+\frac{1}{c^{7}}\left[\frac{G}{r_{1}^{2}}\{\right. \\
& \left(n_{1}, S_{1}, v\right) v^{i} v^{j}\left(-1+3 \nu+\frac{\delta m}{m}(1-\nu)\right)+v^{(i} \varepsilon^{j) k l} S_{1}^{k} v^{l}\left(1-3 \nu+\frac{\delta m}{m}(-1+\nu)\right)\left(n_{1} v\right) \\
& \left.+v^{(i} \varepsilon^{j) k l} n_{1}^{k} S_{1}^{l}\left[\left(-1+3 \nu+\frac{\delta m}{m}(1-3 \nu)\right) v^{2}+\left(3-9 \nu+\frac{\delta m}{m}(-3+3 \nu)\right)\left(n_{1} v\right)^{2}\right]\right\} \\
& +\frac{G^{2} m}{r_{1}^{2} r_{2}}\left\{8 \nu\left(n_{1}, S_{1}, v\right) \delta^{i j}+v^{(i} \varepsilon^{j) k l} n_{1}^{k} S_{1}^{l}\left(-4-8 \nu+4 \frac{\delta m}{m}\right)\right\}+\frac{G^{2} m}{r_{1}^{3}} 4 \nu v^{(i} \varepsilon^{j) k l} n_{1}^{k} S_{1}^{l} \\
& +\frac{G^{2} m}{r r_{1}^{2}} \nu\left\{-8\left(n_{1}, S_{1}, v\right) \delta^{i j}+2 \frac{\delta m}{m}(n v) n^{(i} \varepsilon^{j) k l} n_{1}^{k} S_{1}^{l}+2 \frac{\delta m}{m} v^{(i} \varepsilon^{j) k l} n_{1}^{k} S_{1}^{l}\right\} \\
& +\frac{G^{2} m}{r^{2} r_{2}}\left\{\left(n_{2}, n, S_{1}\right) \delta^{i j}\left(\nu-\nu \frac{\delta m}{m}\right)\left(n_{2} v\right)+\left(n, S_{1}, v\right) \delta^{i j}\left(2-2 \frac{\delta m}{m}\right)\right. \\
& \left.+v^{(i} \varepsilon^{j) k l} n^{k} S_{1}^{l}\left(-4 \nu+4 \nu \frac{\delta m}{m}\right)\right\}+\frac{G^{2} m}{r^{2} r_{1}}\left\{\left(n_{1}, n, S_{1}\right) \delta^{i j}\left(-\nu+\nu \frac{\delta m}{m}\right)\left(n_{1} v\right)\right. \\
& +8 \nu\left(n, S_{1}, v\right) \delta^{i j}+n^{(i} \varepsilon^{j) k l} n_{1}^{k} S_{1}^{l}\left(-2+4 \nu+2 \frac{\delta m}{m}\right)\left(n_{1} v\right)+n^{(i} \varepsilon^{j) k l} S_{1}^{k} v^{l}\left(-4+4 \frac{\delta m}{m}\right) \\
& \left.+v^{(i} \varepsilon^{j) k l} n_{1}^{k} S_{1}^{l}\left(-1+2 \nu+\frac{\delta m}{m}\right)\left(n_{1} n\right)+v^{(i} \varepsilon^{j) k l} n^{k} S_{1}^{l}\left(1-6 \nu+\frac{\delta m}{m}(-1-4 \nu)\right)\right\} \\
& +\frac{G^{2} m}{r^{3}}\left\{\left(n_{1}, n, S_{1}\right) \delta^{i j}\left(-\frac{3}{2}+\frac{3}{2} \frac{\delta m}{m}\right)(n v)+\left(n_{2}, n, S_{1}\right) \delta^{i j}\left(3-3 \frac{\delta m}{m}\right)(n v)\right. \\
& +\left(n_{1}, S_{1}, v\right) \delta^{i j}\left(-\frac{3}{2}+\frac{3}{2} \frac{\delta m}{m}\right)+\left(n_{2}, S_{1}, v\right) \delta^{i j}\left(2-2 \frac{\delta m}{m}\right) \\
& +\left(n, S_{1}, v\right) \delta^{i j}\left[\left(3-3 \frac{\delta m}{m}\right)\left(n_{1} n\right)+\left(-3+3 \frac{\delta m}{m}\right)\left(n_{2} n\right)\right] \\
& \left.+n^{(i} \varepsilon^{j) k l} n_{1}^{k} S_{1}^{l}\left(3-3 \frac{\delta m}{m}\right)(n v)+v^{(i} \varepsilon^{j) k l} n_{1}^{k} S_{1}^{l}\left(-1+\frac{\delta m}{m}\right)\right\} \\
& +G^{2} m\left\{4 \delta^{i j}\left(\underset{1}{\partial_{b}} \underset{2}{\partial_{l a}} g\right) \varepsilon^{k a b} S_{1}^{k} v^{l}\left(-1+\frac{\delta m}{m}\right)-16 \nu \delta^{i j}\left(\underset{1}{\partial_{a b}} \underset{2}{\partial_{a}} g\right) \varepsilon^{k l b} S_{1}^{k} v^{l}\right. \\
& +\delta^{i j}\left(\underset{1}{\partial_{b}} \underset{2}{\partial_{a a}} g\right) \varepsilon^{k l b} S_{1}^{k} v^{l}\left(4-8 \nu-4 \frac{\delta m}{m}\right)+8 \underset{1}{\partial_{k a}} \underset{2}{\partial}(j g) \varepsilon^{i) b a} S_{1}^{b} v^{k}\left(1-\frac{\delta m}{m}\right) \\
& +8\left(\underset{1}{\partial}{ }_{(i a} \underset{2}{\partial_{j}} g\right) \varepsilon^{b k a} S_{1}^{b} v^{k}\left(1-\frac{\delta m}{m}\right)+16 \nu\left(\underset{1}{\partial_{(j a} \underset{2}{\partial} b} g\right) \varepsilon^{k a b} S_{1}^{k} v^{i)} \\
& \left.\left.-16 \nu\left(\underset{1}{\partial_{a b}} \underset{2}{\partial_{a}} g\right) \varepsilon^{(j k b} S_{1}^{k} v^{i)}\right\}\right]+(1 \leftrightarrow 2)+\mathcal{O}\left(\frac{1}{c^{8}}\right) \text {. }
\end{aligned}
$$




\section{COMPACT BINARIES IN QUASI-CIRCULAR ORBITS}

\section{A. Reduction to circular orbits}

We now present our results in the case where the orbit is nearly circular (if one neglects the radiation-reaction effects), i.e. has a constant radius and is planar apart from small perturbations induced by the spins that cause the orbital plane to slowly precess. Most compact binaries will be in quasi-circular orbits when they are observed by the gravitational wave detectors. Assuming this sort of motion drastically simplifies the long expressions of the previous Section, which become immediately much shorter. We may thus include also, for the sake of completeness and convenience, all non-spinning terms up to 3PN order. The results that we display are then complete up to $3.5 \mathrm{PN}$ order and linear order in the spins (remember that we neglect spin-spin contributions) 7

When comparing with the various expressions in the literature for the lower order spinorbit corrections, we have to keep in mind that different definitions of the spin variables yield different numerical coefficients. In particular, as Ref. [28] and Section VI of Ref. [46] did not make use of conserved-norm spins, the results there differ from the ones that we find here at next-to-leading order. By contrast, our energy, angular momentum and precession vector agree with those given in Section VII of [46], where conserved-norm spin variables are introduced, because these spin variables, although constructed differently, turn out to agree with ours when evaluated in the CM frame.

For the kinematics of quasi-circular orbits, we use the same conventions as Ref. [28] which can be summarized as follows. We introduce an orthonormal triad $\{\mathbf{n}, \boldsymbol{\lambda}, \boldsymbol{\ell}\}$, such that $\mathbf{n}=\mathbf{x} / r, \boldsymbol{\ell}=\mathbf{L}_{\mathrm{N}} /\left|\mathbf{L}_{\mathrm{N}}\right|$ where $\mathbf{L}_{\mathrm{N}} \equiv \mu \mathbf{x} \times \mathbf{v}$ denotes the Newtonian angular momentum, and $\boldsymbol{\lambda}=\boldsymbol{\ell} \times \mathbf{n}$. All the relevant CM quantities have been defined in Section IIIA. We project out the spins on the latter orthonormal basis, as $\mathbf{S}=S_{n} \mathbf{n}+S_{\lambda} \boldsymbol{\lambda}+S_{\ell} \boldsymbol{\ell}$ and similarly for $\boldsymbol{\Sigma}$. By definition of quasi-circular orbits, the orbital separation decreases as $\dot{r}=\mathcal{O}\left(1 / c^{5}\right)$, entirely due to the gravitational radiation reaction. The spins contribute to this radiation reaction only at $1.5 \mathrm{PN}$ order beyond the dominant term $\mathcal{O}\left(1 / c^{5}\right)$, i.e. at $4 \mathrm{PN}$ order [45], which is neglected here. We define the orbital frequency $\omega$ and the precession frequency $\varpi$ by the relations $\omega=\boldsymbol{\lambda} \cdot \dot{\boldsymbol{n}}$ and $\varpi=\boldsymbol{\ell} \cdot \dot{\boldsymbol{\lambda}}$, so that the triad evolution equations read $\dot{\boldsymbol{n}}=\omega \boldsymbol{\lambda}$, $\dot{\boldsymbol{\lambda}}=-\omega \boldsymbol{n}+\varpi \boldsymbol{\ell}$ and $\dot{\boldsymbol{\ell}}=-\varpi \boldsymbol{\lambda}$. Then, posing $\varpi=\varpi \boldsymbol{n}$, we have $\dot{\boldsymbol{\ell}}=\varpi \times \boldsymbol{\ell}$ which is the natural definition for the precession vector $\varpi$ of the orbital plane 8 The acceleration may be then written in our moving basis as

$$
\frac{\mathrm{d} \mathbf{v}}{\mathrm{d} t}=-\omega^{2} r \mathbf{n}+(r \dot{\omega}+2 \dot{r} \omega) \boldsymbol{\lambda}+a_{\ell} \boldsymbol{\ell}+\mathcal{O}\left(\frac{1}{c^{8}}\right),
$$

in which we have set $a_{\ell} \equiv r \omega \varpi$ and neglected a term $\ddot{r}=\mathcal{O}\left(1 / c^{8}\right)$. The spin contributions in the term proportional to $\boldsymbol{\lambda}$ are, by the same argument as before, of negligible $4 \mathrm{PN}$ order since the spin terms in $\omega$ come with a factor $1 / c^{3}$ and can thus be regarded as constant at the considered order, see Eq. (4.2) below and the discussion thereafter. As a result, the spins can contribute to the acceleration only through $\omega$ and the quantity $a_{\ell}$ at $3.5 \mathrm{PN}$ order.

\footnotetext{
${ }^{7}$ Note that in order to reduce the results of the previous Section to circular orbits, the contributions from the non-spinning terms up to $2 \mathrm{PN}$ order, which are not presented there, must be taken into account.

${ }^{8}$ We have $\varpi=-\omega_{\text {prec }}$ in the notation of Ref. [51].
} 


\section{B. Equations of motion and precession vector}

In the case of circular orbits, the relativistic generalization of Kepler's law relating the orbital frequency $\omega$ to the body separation $r$, is most conveniently expressed in terms of the post-Newtonian parameter $\gamma \equiv G m /\left(r c^{2}\right)=\mathcal{O}\left(1 / c^{2}\right)$. It is derived by reducing the CM relative acceleration given in Section IIIC. We find

$$
\begin{aligned}
\omega^{2}=\frac{G m}{r^{3}}\{1 & +\gamma(-3+\nu)+\gamma^{2}\left(6+\frac{41}{4} \nu+\nu^{2}\right) \\
& +\gamma^{3}\left(-10+\left[-\frac{75707}{840}+\frac{41}{64} \pi^{2}+22 \ln \left(\frac{r}{r_{0}^{\prime}}\right)\right] \nu+\frac{19}{2} \nu^{2}+\nu^{3}\right) \\
& +\frac{\gamma^{3 / 2}}{G m^{2}}\left[-5 S_{\ell}-3 \frac{\delta m}{m} \Sigma_{\ell}\right]+\frac{\gamma^{5 / 2}}{G m^{2}}\left[\left(\frac{45}{2}-\frac{27}{2} \nu\right) S_{\ell}+\frac{\delta m}{m}\left(\frac{27}{2}-\frac{13}{2} \nu\right) \Sigma_{\ell}\right] \\
& +\frac{\gamma^{7 / 2}}{G m^{2}}\left[\left(-\frac{495}{8}-\frac{561}{8} \nu-\frac{51}{8} \nu^{2}\right) S_{\ell}+\frac{\delta m}{m}\left(-\frac{297}{8}-\frac{341}{8} \nu-\frac{21}{8} \nu^{2}\right) \Sigma_{\ell}\right] \\
& \left.+\mathcal{O}\left(\frac{1}{c^{8}}\right)\right\} .
\end{aligned}
$$

This expression is clearly gauge dependent since it involves the separation $r$ defined in harmonic coordinates. Notably, the presence of a gauge constant $r_{0}^{\prime}$ in the non-spin part at the $3 \mathrm{PN}$ order attests the occurrence of a pure gauge effect which disappears from the observables of the problem (see e.g. [11]). As we stated before, the spin part of $\omega$ is constant at linear order in spin and up to 3.5PN order, for $r$ is constant at the $2 \mathrm{PN}$ approximation and so are the spin components $S_{\ell}^{A}$ (with $A=1,2$ ): indeed, the precession vectors $\Omega_{A}$ are proportional to $\ell$, see Eq. (4.5), hence $\mathrm{d} S_{\ell}^{A} / \mathrm{d} t=-\varpi S_{\lambda}^{A}$, the right-hand side of the latter relation being quadratic in spin since $\varpi$ is proportional to the spin, see Eq. (4.4) below.

When computing physical quantities, it is useful to eliminate the coordinate distance $r$ (or equivalently $\gamma$ ) by reexpressing it in terms of $\omega$ or, more conveniently, of the post-Newtonian frequency-related parameter $x \equiv\left(G m \omega / c^{3}\right)^{2 / 3}$. Inverting Eq. (4.2), we obtain

$$
\begin{aligned}
\gamma=x\{ & +x\left(1-\frac{1}{3} \nu\right)+x^{2}\left(1-\frac{65}{12} \nu\right) \\
& +x^{3}\left(1+\left[-\frac{2203}{2520}-\frac{41}{192} \pi^{2}-\frac{22}{3} \ln \left(\frac{r}{r_{0}^{\prime}}\right)\right] \nu+\frac{229}{36} \nu^{2}+\frac{1}{81} \nu^{3}\right) \\
& +\frac{x^{3 / 2}}{G m^{2}}\left[\frac{5}{3} S_{\ell}+\frac{\delta m}{m} \Sigma_{\ell}\right]+\frac{x^{5 / 2}}{G m^{2}}\left[\left(\frac{10}{3}+\frac{8}{9} \nu\right) S_{\ell}+2 \frac{\delta m}{m} \Sigma_{\ell}\right] \\
& +\frac{x^{7 / 2}}{G m^{2}}\left[\left(5-\frac{127}{12} \nu-6 \nu^{2}\right) S_{\ell}+\frac{\delta m}{m}\left(3-\frac{61}{6} \nu-\frac{8}{3} \nu^{2}\right) \Sigma_{\ell}\right] \\
& \left.+\mathcal{O}\left(\frac{1}{c^{8}}\right)\right\} .
\end{aligned}
$$

For the component of the acceleration along $\boldsymbol{\ell}$, which vanishes in the absence of spins, after inserting the explicit value of $\mathbf{v}=\dot{\boldsymbol{r}} \mathbf{n}+r \omega \boldsymbol{\lambda}$ and eliminating $r$ in terms of $x$ by means of the 
above formula, we find (recall that $\left.a_{\ell}=r \omega \varpi\right)$

$$
\begin{aligned}
a_{\ell}=\frac{c^{4} x^{7 / 2}}{G^{2} m^{3}}\{ & {\left[7 S_{n}+3 \frac{\delta m}{m} \Sigma_{n}\right]+x\left[\left(-10-\frac{29}{3} \nu\right) S_{n}+\frac{\delta m}{m}\left(-6-\frac{9}{2} \nu\right) \Sigma_{n}\right] } \\
& \left.+x^{2}\left[\left(\frac{3}{2}+\frac{59}{4} \nu+\frac{52}{9} \nu^{2}\right) S_{n}+\frac{\delta m}{m}\left(\frac{3}{2}+\frac{73}{8} \nu+\frac{17}{6} \nu^{2}\right) \Sigma_{n}\right]\right\}+\mathcal{O}\left(\frac{1}{c^{8}}\right) .
\end{aligned}
$$

The last quantity that we need in order to complete the evolution equations for circular

orbits is the precession vector $\Omega_{1}$. From Section $\amalg$ IIB, we readily get

$$
\begin{aligned}
\Omega_{1}=\frac{c^{3} x^{5 / 2}}{G m} \ell\{ & \left(\frac{3}{4}+\frac{1}{2} \nu-\frac{3}{4} \frac{\delta m}{m}\right)+x\left[\frac{9}{16}+\frac{5}{4} \nu-\frac{1}{24} \nu^{2}+\frac{\delta m}{m}\left(-\frac{9}{16}+\frac{5}{8} \nu\right)\right] \\
& +x^{2}\left[\frac{27}{32}+\frac{3}{16} \nu-\frac{105}{32} \nu^{2}-\frac{1}{48} \nu^{3}+\frac{\delta m}{m}\left(-\frac{27}{32}+\frac{39}{8} \nu-\frac{5}{32} \nu^{2}\right)\right] \\
& \left.+\mathcal{O}\left(\frac{1}{c^{6}}\right)\right\} .
\end{aligned}
$$

\section{Conserved integrals of the motion}

Let us now provide the expressions of the energy $E$ and of the orbital angular momentum

$\mathbf{L}$ reduced to the case of circular motion. Applying to the CM expression (3.9) the same reduction procedure as for the computation of $a_{\ell}$ in the previous Subsection yields

$$
\begin{aligned}
E=-\frac{\mu c^{2} x}{2}\{ & +x\left(-\frac{3}{4}-\frac{1}{12} \nu\right)+x^{2}\left(-\frac{27}{8}+\frac{19}{8} \nu-\frac{1}{24} \nu^{2}\right) \\
& +x^{3}\left(-\frac{675}{64}+\left[\frac{34445}{576}-\frac{205}{96} \pi^{2}\right] \nu-\frac{155}{96} \nu^{2}-\frac{35}{5184} \nu^{3}\right) \\
& +\frac{x^{3 / 2}}{G m^{2}}\left[\frac{14}{3} S_{\ell}+2 \frac{\delta m}{m} \Sigma_{\ell}\right]+\frac{x^{5 / 2}}{G m^{2}}\left[\left(11-\frac{61}{9} \nu\right) S_{\ell}+\frac{\delta m}{m}\left(3-\frac{10}{3} \nu\right) \Sigma_{\ell}\right] \\
& +\frac{x^{7 / 2}}{G m^{2}}\left[\left(\frac{135}{4}-\frac{367}{4} \nu+\frac{29}{12} \nu^{2}\right) S_{\ell}+\frac{\delta m}{m}\left(\frac{27}{4}-39 \nu+\frac{5}{4} \nu^{2}\right) \Sigma_{\ell}\right] \\
& \left.+\mathcal{O}\left(\frac{1}{c^{8}}\right)\right\},
\end{aligned}
$$

where the non-spin terms are taken from Ref. [11]. Notice that since $E$ is a physical observable, the gauge constant $r_{0}^{\prime}$ has canceled out as expected. For the total angular momentum, we obtain from the CM expression provided in Appendix D:

$$
\begin{aligned}
\mathbf{L}=\frac{G m^{2}}{c x^{1 / 2}} \nu\{\ell[1 & +x\left(\frac{3}{2}+\frac{1}{6} \nu\right)+x^{2}\left(\frac{27}{8}-\frac{19}{8} \nu+\frac{1}{24} \nu^{2}\right) \\
& \left.+x^{3}\left(\frac{135}{16}+\left[-\frac{6889}{144}+\frac{41}{24} \pi^{2}\right] \nu+\frac{31}{24} \nu^{2}+\frac{7}{1296} \nu^{3}\right)\right]
\end{aligned}
$$




$$
\begin{gathered}
+\frac{x^{3 / 2}}{G m^{2}}\left(\boldsymbol{\ell}\left[-\frac{35}{6} S_{\ell}-\frac{5}{2} \frac{\delta m}{m} \Sigma_{\ell}\right]+\boldsymbol{\lambda}\left[-3 S_{\lambda}-\frac{\delta m}{m} \Sigma_{\lambda}\right]+\mathbf{n}\left[\frac{1}{2} S_{n}+\frac{1}{2} \frac{\delta m}{m} \Sigma_{n}\right]\right) \\
+\frac{x^{5 / 2}}{G m^{2}}\left(\boldsymbol{\ell}\left[\left(-\frac{77}{8}+\frac{427}{72} \nu\right) S_{\ell}+\frac{\delta m}{m}\left(-\frac{21}{8}+\frac{35}{12} \nu\right) \Sigma_{\ell}\right]\right. \\
+\boldsymbol{\lambda}\left[\left(-\frac{7}{2}+3 \nu\right) S_{\lambda}+\frac{\delta m}{m}\left(-\frac{1}{2}+\frac{4}{3} \nu\right) \Sigma_{\lambda}\right] \\
\left.+\mathbf{n}\left[\left(\frac{11}{8}-\frac{19}{24} \nu\right) S_{n}+\frac{\delta m}{m}\left(\frac{11}{8}-\frac{5}{12} \nu\right) \Sigma_{n}\right]\right) \\
+\frac{x^{7 / 2}}{G m^{2}}\left(\boldsymbol{\ell}\left[\left(-\frac{405}{16}+\frac{1101}{16} \nu-\frac{29}{16} \nu^{2}\right) S_{\ell}+\frac{\delta m}{m}\left(-\frac{81}{16}+\frac{117}{4} \nu-\frac{15}{16} \nu^{2}\right) \Sigma_{\ell}\right]\right. \\
+\boldsymbol{\lambda}\left[\left(-\frac{29}{4}+\frac{1}{12} \nu-\frac{4}{3} \nu^{2}\right) S_{\lambda}+\frac{\delta m}{m}\left(-\frac{1}{2}-\frac{79}{24} \nu-\frac{2}{3} \nu^{2}\right) \Sigma_{\lambda}\right] \\
\left.+\mathbf{n}\left[\left(\frac{61}{16}-\frac{1331}{48} \nu+\frac{11}{48} \nu^{2}\right) S_{n}+\frac{\delta m}{m}\left(\frac{61}{16}-\frac{367}{24} \nu+\frac{5}{48} \nu^{2}\right) \Sigma_{n}\right]\right) \\
\left.+\mathcal{O}\left(\frac{1}{c^{8}}\right)\right\} \cdot
\end{gathered}
$$

The non-spin parts can be found for instance in Ref. [52]. At the next-to-leading order in spin-orbit effects, for both $E$ and $\mathbf{L}$, we recover the results of Section VII of Ref. [46], which are given in terms of the conserved-norm spin variables defined there.

\section{Metric regularized at a particle location}

Finally, we give the expression of the spin part of the near-zone metric regularized at the location of body 1 for circular orbits:

$$
\begin{aligned}
\left(g_{S} 00\right)_{1}= & \frac{x^{5 / 2}}{G m^{2}}\left[\left(\frac{5}{3}-4 \nu-\frac{5}{3} \frac{\delta m}{m}\right) S_{\ell}+\left(-1+2 \nu+\frac{\delta m}{m}(1-2 \nu)\right) \Sigma_{\ell}\right] \\
& +\frac{x^{7 / 2}}{G m^{2}} \nu\left[\left(\frac{11}{9}+\frac{16}{3} \nu-\frac{56}{9} \frac{\delta m}{m}\right) S_{\ell}+\left(-\frac{7}{2}+\frac{20}{3} \nu+\frac{\delta m}{m}\left(\frac{1}{2}+\frac{8}{3} \nu\right)\right) \Sigma_{\ell}\right] \\
& +\frac{x^{9 / 2}}{G m^{2}} \nu\left[\left(\frac{9}{4}+\frac{101}{3} \nu-\frac{8}{3} \nu^{2}+\frac{\delta m}{m}\left(-\frac{45}{2}+\frac{118}{3} \nu\right)\right) S_{\ell}\right. \\
& \left.+\left(-\frac{13}{8}+\frac{1009}{30} \nu-64 \nu^{2}+\frac{\delta m}{m}\left(-\frac{41}{8}+\frac{79}{6} \nu-\frac{4}{3} \nu^{2}\right)\right) \Sigma_{\ell}\right] \\
& +\mathcal{O}\left(\frac{1}{c^{10}}\right), \\
\left(g_{S}^{g}{ }_{0 i}\right)_{1}= & \frac{x^{2}}{G m^{2}}\left(\ell^{i}\left[\left(1-\frac{\delta m}{m}\right) S_{\lambda}+2 \nu \Sigma_{\lambda}\right]+\lambda^{i}\left[\left(-1+\frac{\delta m}{m}\right) S_{\ell}-2 \nu \Sigma_{\ell}\right]\right)
\end{aligned}
$$




$$
\begin{aligned}
& +\frac{x^{3}}{G m^{2}}\left(\ell^{i}\left[\left(1-\frac{1}{6} \nu+\frac{\delta m}{m}\left(-1+\frac{7}{6} \nu\right)\right) S_{\lambda}+\left(\frac{5}{2} \nu-\frac{7}{3} \nu^{2}-\frac{3}{2} \nu \frac{\delta m}{m}\right) \Sigma_{\lambda}\right]\right. \\
& \left.+\lambda^{i}\left[\left(-1-\frac{4}{3} \nu+\frac{\delta m}{m}\left(1-\frac{8}{3} \nu\right)\right) S_{\ell}+\left(-4 \nu+\frac{16}{3} \nu^{2}\right) \Sigma_{\ell}\right]\right) \\
& +\frac{x^{7 / 2}}{G m^{2}} \nu\left(-\frac{2}{3} \Sigma_{\ell} n^{i}+\frac{2}{3} \Sigma_{n} \ell^{i}\right) \\
& +\frac{x^{4}}{G m^{2}}\left(\ell ^ { i } \left[\left(1+\frac{69}{8} \nu-\frac{649}{72} \nu^{2}+\frac{\delta m}{m}\left(-1+\frac{25}{8} \nu-\frac{35}{72} \nu^{2}\right)\right) S_{\lambda}\right.\right. \\
& \left.+\left(\frac{143}{40} \nu-4 \nu^{2}+\frac{35}{36} \nu^{3}+\frac{\delta m}{m}\left(\frac{23}{40} \nu-\frac{7}{4} \nu^{2}\right)\right) \Sigma_{\lambda}\right] \\
& +\lambda^{i}\left[\left(-1-\frac{23}{4} \nu+\frac{389}{9} \nu^{2}+\frac{\delta m}{m}\left(1-\frac{25}{4} \nu+\frac{19}{9} \nu^{2}\right)\right) S_{\ell}\right. \\
& \left.\left.+\left(-\frac{97}{10} \nu+\frac{21}{2} \nu^{2}-\frac{38}{9} \nu^{3}+\frac{\delta m}{m}\left(\frac{13}{10} \nu+16 \nu^{2}\right)\right) \Sigma_{\ell}\right]\right) \\
& +\mathcal{O}\left(\frac{1}{c^{9}}\right) \\
& \left(g_{S}\right)_{1}=\frac{x^{5 / 2}}{G m^{2}}\left(\delta^{i j}\left[\left(\frac{5}{3}-\frac{5}{3} \frac{\delta m}{m}\right) S_{\ell}+\left(-1+4 \nu+\frac{\delta m}{m}\right) \Sigma_{\ell}\right]\right. \\
& \left.+\ell^{(i} \lambda^{j)} \nu\left[4 S_{\lambda}+\left(2+2 \frac{\delta m}{m}\right) \Sigma_{\lambda}\right]+\lambda^{i} \lambda^{j} \nu\left(-2 \Sigma_{\ell}-4 S_{\ell}-2 \Sigma_{\ell} \frac{\delta m}{m}\right)\right) \\
& +\frac{x^{7 / 2}}{G m^{2}}\left(n^{i} n^{j}\left[\left(\frac{5}{3}+14 \nu-\frac{5}{3} \frac{\delta m}{m}\right) S_{\ell}+\left(-1+6 \nu+\frac{\delta m}{m}(1+4 \nu)\right) \Sigma_{\ell}\right]\right. \\
& +\delta^{i j}\left[\left(5-\frac{145}{9} \nu+\frac{\delta m}{m}\left(-5-\frac{8}{9} \nu\right)\right) S_{\ell}+\left(-3+\frac{19}{2} \nu+\frac{\delta m}{m}\left(3-\frac{17}{2} \nu\right)\right) \Sigma_{\ell}\right] \\
& +\ell^{(i} \lambda^{j)} \nu\left[\left(-17-\frac{10}{3} \nu+\frac{\delta m}{m}\right) S_{\lambda}+\left(2+\frac{13}{3} \nu+\frac{\delta m}{m}\left(-10-\frac{5}{3} \nu\right)\right) \Sigma_{\lambda}\right] \\
& +\lambda^{i} \lambda^{j} \nu\left[\left(\frac{38}{3}+\frac{16}{3} \nu-\frac{16}{3} \frac{\delta m}{m}\right) S_{\ell}+\left(-5+\frac{20}{3} \nu+\frac{\delta m}{m}\left(7+\frac{8}{3} \nu\right)\right) \Sigma_{\ell}\right] \\
& \left.+\ell^{(i} n^{j)} \nu\left[68 S_{n}+\left(6+34 \frac{\delta m}{m}\right) \Sigma_{n}\right]\right)+\mathcal{O}\left(\frac{1}{c^{8}}\right) .
\end{aligned}
$$

\section{CONCLUSIONS}

Building on previous work (Ref. [39], referred to as Paper I) we have presented complete results valid at the next-to-next-to-leading order, corresponding to 3.5PN order, for the spin-

orbit contributions to the dynamics of spinning compact binaries. We notably obtained the 
spin-orbit contribution to the evolution (or precession) equations for the spins, the conserved integrals of the motion associated with the Poincaré invariance of the equations (energy, angular momentum and center-of-mass integral), and the near-zone metric regularized at the location of the particles. We also obtained the bulk metric (i.e. at any field point in the near-zone) but with less precision in the 00 and $0 i$ components. The results are given for general orbits in the frame of the binary's center-of-mass, and reduced for quasicircular orbits, which is the mostly relevant case for realistic compact binaries detected by gravitational waves. We systematically make use of a precise definition of the spin variables, which have a conserved Euclidean norm.

The metric in the near zone is especially of interest in applications such as the numerical study of a possible astrophysical environment surrounding black-hole binaries with spins [41], and the comparison with numerical computations of the gravitational self force acting on a particle orbiting a Kerr black hole, which are based on first (or second) order black hole perturbation theory [42, 43]. More generally, all the present analytical results (given explicitly as they are) should be useful for the accurate comparisons between PN predictions and the full numerical computations of the coalescence of two spinning black holes.

The next step in this current program will be to obtain the next-to-next-to-leading order spin-orbit contributions in the gravitational-wave phasing of spinning compact binaries. The GW phasing including high-order spin effects (and especially high-order spin-orbit effects) is of crucial importance for the parameter estimation of signals received from compact binaries by the networks of ground-based as well as, in the future, space-based GW detectors.

\section{ACKNOWLEDGEMENTS}

A.B. is grateful for the support of the European Union FEDER funds, the Spanish Ministry of Economy and Competitiveness project FPA2010-16495 and the Conselleria d'Economia Hisenda i Innovacio of the Govern de les Illes Balears.

\section{Appendix A: Comparison with the ADM formalism}

In this Appendix, we give the 3PN correspondence between our spin variables and those of Ref. [40]. The 2PN correspondence had been investigated in [29]. In Section VII D of Paper I, we showed that our results for the equations of motion were equivalent to the ones obtained there in ADM variables, and we constructed the extension of the contact transformation required to relate the two methods, as well as the $2 \mathrm{PN}$ link between the ADM spin variables and the harmonic-coordinates spin tensor. We shall use here the same notations, with an overbar for all ADM quantities as well as the convenient short-cut $\bar{\pi} \equiv \bar{p} / m$. Let us now extend this correspondence in the dynamics to the $3 \mathrm{PN}$ spin precession equations.

The ADM conserved-norm vector $\overline{\mathbf{S}}$ obeys the precession equation

$$
\frac{\mathrm{d} \overline{\mathbf{S}}}{\mathrm{d} t}=\overline{\mathbf{\Omega}} \times \overline{\mathbf{S}}
$$

where the precession vector $\bar{\Omega}$ can be read directly on the spin-orbit Hamiltonian $H_{\mathrm{SO}}=$ $\bar{\Omega}_{1} \cdot \overline{\mathbf{S}}_{1}+\bar{\Omega}_{2} \cdot \overline{\mathbf{S}}_{2}$ in Ref. [29]. The two conserved-norm spins start differing at $2 \mathrm{PN}$ order, 
and from the fact that they must have the same magnitude we may write

$$
\mathbf{S}(\overline{\mathbf{x}}, \overline{\mathbf{p}})=\overline{\mathbf{S}}+\boldsymbol{\theta}(\overline{\mathbf{x}}, \overline{\mathbf{p}}) \times \overline{\mathbf{S}}+\mathcal{O}\left(\frac{1}{c^{8}}\right)
$$

where $\boldsymbol{\theta}$ represents a small rotation vector starting at $\mathcal{O}\left(1 / c^{4}\right)$. Its expression is found by comparing the precession equations for the two spin variables. Indeed, if we plug this relation into the precession equation for $\mathbf{S}$, noticing that $\boldsymbol{\Omega}(\overline{\mathbf{x}}, \overline{\mathbf{p}})=\overline{\mathbf{\Omega}}+\mathcal{O}\left(1 / c^{4}\right)$, we obtain

$$
\boldsymbol{\Omega} \times \overline{\mathbf{S}}=\left(\overline{\boldsymbol{\Omega}}+\frac{\mathrm{d} \boldsymbol{\theta}}{\mathrm{d} t}+\boldsymbol{\theta} \times \overline{\boldsymbol{\Omega}}\right) \times \overline{\mathbf{S}}+\mathcal{O}\left(\frac{1}{c^{8}}\right) .
$$

Since we are looking for an expression of $\boldsymbol{\theta}$ that does not feature any spin variable, we can forget about the degeneracy remaining along the direction of $\overline{\mathbf{S}}$ and we write

$$
\frac{\mathrm{d} \boldsymbol{\theta}}{\mathrm{d} t}+\boldsymbol{\theta} \times \overline{\boldsymbol{\Omega}}=\boldsymbol{\Omega}(\overline{\mathbf{x}}, \overline{\mathbf{p}})-\overline{\boldsymbol{\Omega}}+\mathcal{O}\left(\frac{1}{c^{8}}\right) .
$$

Using the method of undetermined coefficients for the dimensionless vector $\boldsymbol{\theta}$, we obtain a unique solution (for particle 1),

$$
\boldsymbol{\theta}_{1}=\frac{1}{c^{4}} \boldsymbol{\theta}_{1}^{2 \mathrm{PN}}+\frac{1}{c^{6}} \boldsymbol{\theta}_{1}^{3 \mathrm{PN}}+\mathcal{O}\left(\frac{1}{c^{8}}\right)
$$

with the following expressions for the $2 \mathrm{PN}$ and $3 \mathrm{PN}$ contributions $: 9$

$$
\begin{aligned}
& \boldsymbol{\theta}_{1}^{2 \mathrm{PN}}=\frac{G m_{2}}{\bar{r}_{12}}\left[-\frac{1}{4} \overline{\mathbf{n}}_{12} \times \overline{\boldsymbol{\pi}}_{1}\left(\bar{n}_{12} \bar{\pi}_{2}\right)+\overline{\mathbf{n}}_{12} \times \overline{\boldsymbol{\pi}}_{2}\left(\bar{n}_{12} \bar{\pi}_{2}\right)-\frac{1}{4} \overline{\boldsymbol{\pi}}_{1} \times \overline{\boldsymbol{\pi}}_{2}\right], \\
& \left.\boldsymbol{\theta}_{1}^{3 \mathrm{PN}}=\frac{G}{\bar{r}_{12}}{ }^{(3)} \boldsymbol{\theta}_{1}^{0,1} m_{2}+\frac{G^{2}}{\bar{r}_{12}^{2}}{ }^{\left({ }^{(3)}\right.} \boldsymbol{\theta}_{1}^{1,1} m_{1} m_{2}+{ }^{(3)} \boldsymbol{\theta}_{1}^{0,2} m_{2}^{2}\right] \text {, } \\
& { }^{(3)} \boldsymbol{\theta}_{1}^{0,1}=\overline{\mathbf{n}}_{12} \times \overline{\boldsymbol{\pi}}_{1}\left[\frac{1}{16} \bar{\pi}_{1}^{2}\left(\bar{n}_{12} \bar{\pi}_{2}\right)+\frac{9}{16}\left(\bar{n}_{12} \bar{\pi}_{2}\right)^{3}-\frac{5}{16} \bar{\pi}_{2}^{2}\left(\bar{n}_{12} \bar{\pi}_{2}\right)\right] \\
& +\overline{\mathbf{n}}_{12} \times \overline{\boldsymbol{\pi}}_{2}\left[-\frac{3}{4}\left(\bar{n}_{12} \bar{\pi}_{1}\right)\left(\bar{n}_{12} \bar{\pi}_{2}\right)^{2}-\frac{3}{4}\left(\bar{n}_{12} \bar{\pi}_{2}\right)^{3}-\frac{1}{2}\left(\bar{n}_{12} \bar{\pi}_{2}\right)\left(\bar{\pi}_{1} \bar{\pi}_{2}\right)\right. \\
& \left.+\frac{1}{4}\left(\bar{n}_{12} \bar{\pi}_{1}\right) \bar{\pi}_{2}^{2}+\frac{1}{4}\left(\bar{n}_{12} \bar{\pi}_{2}\right) \bar{\pi}_{2}^{2}\right] \\
& +\bar{\pi}_{1} \times \bar{\pi}_{2}\left[\frac{1}{16} \bar{\pi}_{1}^{2}+\frac{13}{16}\left(\bar{n}_{12} \bar{\pi}_{2}\right)^{2}+\frac{1}{2}\left(\bar{\pi}_{1} \bar{\pi}_{2}\right)-\frac{9}{16} \bar{\pi}_{2}^{2}\right], \\
& { }^{(3)} \boldsymbol{\theta}_{1}^{1,1}=\overline{\mathbf{n}}_{12} \times \overline{\boldsymbol{\pi}}_{1}\left[\frac{3}{8}\left(\bar{n}_{12} \bar{\pi}_{1}\right)-\left(\bar{n}_{12} \bar{\pi}_{2}\right)\right]+\overline{\mathbf{n}}_{12} \times \overline{\boldsymbol{\pi}}_{2}\left[-\frac{5}{2}\left(\bar{n}_{12} \bar{\pi}_{1}\right)+\frac{1}{2}\left(\bar{n}_{12} \bar{\pi}_{2}\right)\right]+\frac{35}{16} \overline{\boldsymbol{\pi}}_{1} \times \overline{\boldsymbol{\pi}}_{2}, \\
& { }^{(3)} \boldsymbol{\theta}_{1}^{0,2}=\overline{\mathbf{n}}_{12} \times \overline{\boldsymbol{\pi}}_{1}\left[-\frac{1}{8}\left(\bar{n}_{12} \bar{\pi}_{1}\right)+\frac{3}{2}\left(\bar{n}_{12} \bar{\pi}_{2}\right)\right]-\frac{41}{8} \overline{\mathbf{n}}_{12} \times \overline{\boldsymbol{\pi}}_{2}\left(\bar{n}_{12} \bar{\pi}_{2}\right)+\frac{3}{4} \overline{\boldsymbol{\pi}}_{1} \times \overline{\boldsymbol{\pi}}_{2} .
\end{aligned}
$$

The existence of such a solution for $\boldsymbol{\theta}$ shows also that the 3PN precession equations are equivalent in the two formalisms, which gives us further confidence in all results.

${ }^{9}$ Notice that the $2 \mathrm{PN}$ terms are different from equation (6.4) in [29], since they compare the ADM spin variable to the different conserved variable $\mathbf{S}_{\mathrm{BBF}}^{c}$ used in Ref. [46]. 


\section{Appendix B: Conversion from the conserved spin vector to the spin tensor}

In this Section we use the same notations for the orbital variables as in Paper I, except for the spin tensor, denoted here $\tilde{S}^{i j}$. From Eqs. (2.2) and (2.15), we obtain for our conserved norm spin vector, expressed in terms of the spin tensor $\tilde{S}^{i j}$, the following expressions:

$$
\begin{aligned}
\mathbf{S}_{1}= & \frac{1}{2} \varepsilon^{i j k} \tilde{S}_{1}^{j k}+\frac{1}{c^{2}} \mathbf{S}_{1}^{1 \mathrm{PN}}+\frac{1}{c^{4}} \mathbf{S}_{1}^{2 \mathrm{PN}}+\frac{1}{c^{6}} \mathbf{S}_{1}^{3 \mathrm{PN}}+\mathcal{O}\left(1 / c^{8}\right) \\
\mathbf{S}_{1}^{1 \mathrm{PN}}= & { }^{(1)} \mathbf{S}_{1}^{0,0}+\frac{G}{r_{12}}{ }^{(1)} \mathbf{S}_{\mathbf{1}}{ }^{0,1} m_{2} \\
\mathbf{S}_{1}^{2 \mathrm{PN}}= & { }^{(2)} \mathbf{S}_{1}^{0,0}+\frac{G}{r_{12}}{ }^{(2)} \mathbf{S}_{1}^{0,1} m_{2}+\frac{G^{2}}{r_{12}^{2}}\left[{ }^{(2)} \mathbf{S}_{1}^{1,1} m_{1} m_{2}+{ }^{(2)} \mathbf{S}_{1}^{0,2} m_{2}^{2}\right] \\
\mathbf{S}_{\mathbf{1}}{ }^{3 \mathrm{PN}}= & { }^{(3)} \mathbf{S}_{\mathbf{1}}{ }^{0,0}+\frac{G}{r_{12}}{ }^{(3)} \mathbf{S}_{1}^{0,1} m_{2}+\frac{G^{2}}{r_{12}^{2}}\left[{ }^{(3)} \mathbf{S}_{1}^{1,1} m_{1} m_{2}+{ }^{(3)} \mathbf{S}_{1}^{0,2} m_{2}^{2}\right] \\
& +\frac{G^{3}}{r_{12}^{3}}\left[{ }^{(3)} \mathbf{S}_{1}^{2,1} m_{1}^{2} m_{2}+{ }^{(3)} \mathbf{S}_{1}^{1,2} m_{1} m_{2}^{2}+{ }^{(3)} \mathbf{S}_{1}^{0,3} m_{2}^{3}\right]
\end{aligned}
$$

with, using like in Paper I the notation $(\varepsilon a \tilde{S})=\varepsilon^{i j k} a^{i} \tilde{S}^{j k}$ :

$$
\begin{aligned}
\left({ }^{(1)} S_{1}^{0,0}\right)^{i}= & \frac{1}{4} v_{1}^{2} \varepsilon^{i j k} \tilde{S}_{1}^{j k}-\frac{1}{4}\left(\varepsilon v_{1} \tilde{S}_{1}\right) v_{1}^{i}-\varepsilon^{i j k} v_{1}^{j} v_{1}^{l} \tilde{S}_{1}^{l k}, \\
\left({ }^{(1)} S_{1}^{0,1}\right)^{i}= & \varepsilon^{i j k} \tilde{S}_{1}^{j k}, \\
\left({ }^{(2)} S_{1}^{0,0}\right)^{i}= & \frac{3}{16} v_{1}^{4} \varepsilon^{i j k} \tilde{S}_{1}^{j k}-\frac{3}{16} v_{1}^{2}\left(\varepsilon v_{1} \tilde{S}_{1}\right) v_{1}^{i}-\frac{1}{2} v_{1}^{2} \varepsilon^{i j k} v_{1}^{j} v_{1}^{l} \tilde{S}_{1}^{l k}, \\
\left({ }^{(2)} S_{1}^{0,1}\right)^{i}= & \varepsilon^{i j k} \tilde{S}_{1}^{j k}\left[-\frac{1}{2}\left(n_{12} v_{2}\right)^{2}+\frac{3}{2} v_{1}^{2}-2\left(v_{1} v_{2}\right)+v_{2}^{2}\right]-\frac{3}{2}\left(\varepsilon v_{1} \tilde{S}_{1}\right) v_{1}^{i}+\left(\varepsilon v_{1} \tilde{S}_{1}\right) v_{2}^{i} \\
+ & \left(\varepsilon v_{2} \tilde{S}_{1}\right) v_{1}^{i}-\left(\varepsilon v_{2} \tilde{S}_{1}\right) v_{2}^{i}-6 \varepsilon^{i j k} v_{1}^{j} v_{1}^{l} \tilde{S}_{1}^{l k}+4 \varepsilon^{i j k} v_{1}^{j} v_{2}^{l} \tilde{S}_{1}^{l k} \\
\left({ }^{(2)} S_{1}^{1,1}\right)^{i}= & -\frac{3}{2} \varepsilon^{i j k} \tilde{S}_{1}^{j k}+2\left(\varepsilon n_{12} \tilde{S}_{1}\right) n_{12}^{i}, \\
\left({ }^{(2)} S_{1}^{0,2}\right)^{i}= & \frac{3}{4} \varepsilon^{i j k} \tilde{S}_{1}^{j k}-\frac{1}{4}\left(\varepsilon n_{12} \tilde{S}_{1}\right) n_{12}^{i}, \\
\left({ }^{(3)} S_{1}^{0,0}\right)^{i}= & \frac{5}{32} v_{1}^{6} \varepsilon^{i j k} \tilde{S}_{1}^{j k}-\frac{5}{32} v_{1}^{4}\left(\varepsilon v_{1} \tilde{S}_{1}\right) v_{1}^{i}-\frac{3}{8} v_{1}^{4} \varepsilon^{i j k} v_{1}^{j} v_{1}^{l} \tilde{S}_{1}^{l k}, \\
\left({ }^{(3)} S_{1}^{0,1}\right)^{i}= & \varepsilon^{i j k} \tilde{S}_{1}^{j k}\left[\frac{3}{8}\left(n_{12} v_{2}\right)^{4}-\frac{3}{4}\left(n_{12} v_{2}\right)^{2} v_{1}^{2}+\frac{15}{8} v_{1}^{4}+\left(n_{12} v_{2}\right)^{2}\left(v_{1} v_{2}\right)-3 v_{1}^{2}\left(v_{1} v_{2}\right)+\left(v_{1} v_{2}\right)^{2}\right. \\
& \left.-\left(n_{12} v_{2}\right)^{2} v_{2}^{2}+\frac{3}{2} v_{1}^{2} v_{2}^{2}-2\left(v_{1} v_{2}\right) v_{2}^{2}+v_{2}^{4}\right]+\left(\varepsilon v_{2} \tilde{S}_{1}\right) v_{2}^{i}\left[\frac{1}{2}\left(n_{12} v_{2}\right)^{2}-\frac{1}{2} v_{1}^{2}-v_{2}^{2}\right] \\
& \left.+\frac{1}{4}\left(v_{1} v_{2}\right)+v_{2}^{2}\right]+\left(\varepsilon v_{2} \tilde{S}_{1}\right) v_{1}^{i}\left[-\frac{1}{2}\left(n_{12} v_{2}\right)^{2}+\frac{3}{4} v_{1}^{2}-\frac{1}{4}\left(v_{1} v_{2}\right)+v_{2}^{2}\right] \\
+ & \left(\varepsilon v_{1} \tilde{S}_{1}\right) v_{1}^{i}\left[\frac{3}{4}\left(n_{12} v_{2}\right)^{2}-\frac{15}{8} v_{1}^{2}+\frac{3}{2}\left(v_{1} v_{2}\right)-\frac{3}{2} v_{2}^{2}\right]+\left(\varepsilon v_{1} \tilde{S}_{1}\right) v_{2}^{i}\left[-\frac{1}{2}\left(n_{12} v_{2}\right)^{2}+\frac{3}{4} v_{1}^{2}\right. \\
& \left.3\left(n_{12} v_{2}\right)^{2}-5 v_{1}^{2}+8\left(v_{1} v_{2}\right)-6 v_{2}^{2}\right]+2\left(\varepsilon^{j k l} v_{1}^{j} v_{2}^{k} v_{1}^{m} \tilde{S}_{1}^{m l}\right) v_{1}^{i} \\
& \\
&
\end{aligned}
$$




$$
\begin{aligned}
& +\varepsilon^{i j k} v_{1}^{j} v_{2}^{l} \tilde{S}_{1}^{l k}\left[-2\left(n_{12} v_{2}\right)^{2}+2 v_{1}^{2}-4\left(v_{1} v_{2}\right)+4 v_{2}^{2}\right]-2\left(\varepsilon^{j k l} v_{1}^{j} v_{2}^{k} v_{1}^{m} \tilde{S}_{1}^{m l}\right) v_{2}^{i}, \\
& \left({ }^{(3)} S_{1}^{1,1}\right)^{i}=\varepsilon^{i j k} \tilde{S}_{1}^{j k}\left[\frac{1}{8}\left(n_{12} v_{1}\right)^{2}+\frac{7}{4}\left(n_{12} v_{1}\right)\left(n_{12} v_{2}\right)+\frac{1}{8}\left(n_{12} v_{2}\right)^{2}-\frac{3}{8} v_{1}^{2}-\frac{3}{4}\left(v_{1} v_{2}\right)+\frac{3}{8} v_{2}^{2}\right] \\
& +\left(\varepsilon n_{12} \tilde{S}_{1}\right) n_{12}^{i}\left[4\left(n_{12} v_{1}\right)^{2}-8\left(n_{12} v_{1}\right)\left(n_{12} v_{2}\right)+4 v_{1}^{2}-6\left(v_{1} v_{2}\right)+3 v_{2}^{2}\right] \\
& +\left(\varepsilon n_{12} \tilde{S}_{1}\right) v_{1}^{i}\left[-7\left(n_{12} v_{1}\right)+\frac{9}{2}\left(n_{12} v_{2}\right)\right]+\left(\varepsilon n_{12} \tilde{S}_{1}\right) v_{2}^{i}\left[\frac{15}{2}\left(n_{12} v_{1}\right)-\frac{9}{2}\left(n_{12} v_{2}\right)\right] \\
& +\left(\varepsilon v_{1} \tilde{S}_{1}\right) n_{12}^{i}\left[-7\left(n_{12} v_{1}\right)+\frac{9}{2}\left(n_{12} v_{2}\right)\right]+\left(\varepsilon v_{2} \tilde{S}_{1}\right) n_{12}^{i}\left[\frac{15}{2}\left(n_{12} v_{1}\right)-\frac{9}{2}\left(n_{12} v_{2}\right)\right] \\
& +\frac{13}{4}\left(\varepsilon v_{1} \tilde{S}_{1}\right) v_{1}^{i}-\frac{5}{2}\left(\varepsilon v_{1} \tilde{S}_{1}\right) v_{2}^{i}-\frac{5}{2}\left(\varepsilon v_{2} \tilde{S}_{1}\right) v_{1}^{i}+\frac{5}{2}\left(\varepsilon v_{2} \tilde{S}_{1}\right) v_{2}^{i}-4\left(\varepsilon^{j k l} n_{12}^{j} v_{1}^{k} v_{1}^{m} \tilde{S}_{1}^{m l}\right) n_{12}^{i} \\
& +\varepsilon^{i j k} v_{1}^{j} n_{12}^{l} \tilde{S}_{1}^{l k}\left[-2\left(n_{12} v_{1}\right)-2\left(n_{12} v_{2}\right)\right]+\varepsilon^{i j k} v_{1}^{j} v_{1}^{l} \tilde{S}_{1}^{l k}+2 \varepsilon^{i j k} v_{1}^{j} v_{2}^{l} \tilde{S}_{1}^{l k}, \\
& \left({ }^{(3)} S_{1}^{0,2}\right)^{i}=\varepsilon^{i j k} \tilde{S}_{1}^{j k}\left[\frac{1}{4}\left(n_{12} v_{1}\right)^{2}-\frac{1}{2}\left(n_{12} v_{1}\right)\left(n_{12} v_{2}\right)-\frac{1}{2}\left(n_{12} v_{2}\right)^{2}+\frac{33}{8} v_{1}^{2}-\frac{15}{2}\left(v_{1} v_{2}\right)+\frac{15}{4} v_{2}^{2}\right] \\
& +\left(\varepsilon n_{12} \tilde{S}_{1}\right) n_{12}^{i}\left[\frac{1}{2}\left(n_{12} v_{2}\right)^{2}-\frac{1}{8} v_{1}^{2}\right]+\left(\varepsilon n_{12} \tilde{S}_{1}\right) v_{1}^{i}\left[-\frac{1}{16}\left(n_{12} v_{1}\right)+\frac{1}{4}\left(n_{12} v_{2}\right)\right] \\
& -\frac{1}{4}\left(n_{12} v_{2}\right)\left(\varepsilon n_{12} \tilde{S}_{1}\right) v_{2}^{i}+\left(\varepsilon v_{1} \tilde{S}_{1}\right) n_{12}^{i}\left[-\frac{1}{16}\left(n_{12} v_{1}\right)+\frac{1}{4}\left(n_{12} v_{2}\right)\right]-\frac{33}{8}\left(\varepsilon v_{1} \tilde{S}_{1}\right) v_{1}^{i} \\
& +\frac{15}{4}\left(\varepsilon v_{1} \tilde{S}_{1}\right) v_{2}^{i}-\frac{1}{4}\left(n_{12} v_{2}\right)\left(\varepsilon v_{2} \tilde{S}_{1}\right) n_{12}^{i}+\frac{15}{4}\left(\varepsilon v_{2} \tilde{S}_{1}\right) v_{1}^{i}-\frac{15}{4}\left(\varepsilon v_{2} \tilde{S}_{1}\right) v_{2}^{i}+15 \varepsilon^{i j k} v_{1}^{j} v_{2}^{l} \tilde{S}_{1}^{l k} \\
& +\frac{1}{2}\left(\varepsilon^{j k l} n_{12}^{j} v_{1}^{k} v_{1}^{m} \tilde{S}_{1}^{m l}\right) n_{12}^{i}+\varepsilon^{i j k} v_{1}^{j} n_{12}^{l} \tilde{S}_{1}^{l k}\left[-\left(n_{12} v_{1}\right)+\left(n_{12} v_{2}\right)\right]-\frac{33}{2} \varepsilon^{i j k} v_{1}^{j} v_{1}^{l} \tilde{S}_{1}^{l k} \\
& \left({ }^{(3)} S_{1}^{2,1}\right)^{i}=\frac{5}{2} \varepsilon^{i j k} \tilde{S}_{1}^{j k}-7\left(\varepsilon n_{12} \tilde{S}_{1}\right) n_{12}^{i}, \\
& \left({ }^{(3)} S_{1}^{1,2}\right)^{i}=\frac{17}{6} \varepsilon^{i j k} \tilde{S}_{1}^{j k}-\frac{3}{4}\left(\varepsilon n_{12} \tilde{S}_{1}\right) n_{12}^{i} ， \\
& \left({ }^{(3)} S_{1}^{0,3}\right)^{i}=\frac{1}{2} \varepsilon^{i j k} \tilde{S}_{1}^{j k}-\frac{1}{2}\left(\varepsilon n_{12} \tilde{S}_{1}\right) n_{12}^{i} .
\end{aligned}
$$

As stated above, these conserved spin variables turn out to be the same as the conserved spin vectors of [46], at $2 \mathrm{PN}$ order, when evaluated in the center-of-mass frame.

\section{Appendix C: Center-of-mass position}

In a general frame, the expressions of the whole set of conserved integrals of motion are too lengthy and we therefore chose to present them directly reduced to the center-of-mass frame. However, in this Appendix, we provide the complete expression for the center-of-mass position $\mathbf{G}$ (necessarily in a general frame) since this might be used in the future to reduce higher order results to the center of mass. Denoting the PN expansion of $\mathbf{G}$ in the form

$$
\mathbf{G}=\mathbf{G}_{\mathrm{N}}+\frac{1}{c^{2}} \mathbf{G}_{1 \mathrm{PN}}+\frac{1}{c^{3}} \mathbf{G}_{1.5 \mathrm{PN}}+\frac{1}{c^{4}}\left[\mathbf{G}_{2 \mathrm{PN}}+\mathbf{G}_{S S} 2 \mathrm{PN}\right]+\frac{1}{c^{5}} \mathbf{G}_{2.5 \mathrm{PN}}
$$




$$
+\frac{1}{c^{6}}\left[\mathbf{G}_{3 \mathrm{PN}}+\mathbf{G}_{S S} 3 \mathrm{PN}\right]+\frac{1}{c^{7}} \mathbf{G}_{S} 3 \mathrm{PN}+\mathcal{O}\left(\frac{1}{c^{8}}\right)
$$

we have for the leading and next-to-leading order spin-orbit contributions

$$
\begin{aligned}
& \mathrm{G}_{S} 1.5 \mathrm{PN}=-\mathbf{S}_{\mathbf{1}} \times \mathbf{v}_{\mathbf{1}}+1 \leftrightarrow 2, \\
& \mathrm{G}_{S} 2.5 \mathrm{PN}={ }^{(2.5)} \mathbf{g}_{0,0}+\frac{G}{r_{12}}{ }^{(2.5)} \mathbf{g}_{0,1} m_{2}+1 \leftrightarrow 2,
\end{aligned}
$$

with

$$
\begin{aligned}
{ }^{(2.5)} \mathbf{g}_{0,0} & =-\frac{1}{2} v_{1}^{2} \mathbf{S}_{1} \times \mathbf{v}_{1} \\
{ }^{(2.5)} \mathbf{g}_{0,1} & =\frac{3}{4}\left(n_{12}, S_{1}, v_{1}\right) \mathbf{n}_{12}-\left(n_{12}, S_{1}, v_{2}\right) \mathbf{n}_{12}-\frac{3}{4}\left(n_{12}, S_{1}, v_{1}\right) \tilde{\mathbf{y}}_{1}+2\left(n_{12}, S_{1}, v_{2}\right) \tilde{\mathbf{y}}_{1} \\
& -\frac{1}{4}\left(n_{12}, S_{1}, v_{1}\right) \tilde{\mathbf{y}}_{2}-2\left(n_{12}, S_{1}, v_{2}\right) \tilde{\mathbf{y}}_{2}-2 \mathbf{S}_{1} \times \mathbf{v}_{1}+2 \mathbf{S}_{1} \times \mathbf{v}_{2} \\
& +\mathbf{n}_{12} \times \mathbf{S}_{1}\left[-\left(n_{12} v_{1}\right)-\left(n_{12} v_{2}\right)\right],
\end{aligned}
$$

where we have defined for convenience $\tilde{\mathbf{y}}_{A}=\mathbf{y}_{A} / r_{12}$. Let us emphasize again that these two contributions are the only ones required to derive Eq. (3.3), i.e. to perform our center of mass reduction. In addition to these, the next-to-next-to-leading correction is given by

$$
\mathbf{G}_{S} 3.5 \mathrm{PN}={ }^{(3.5)} \mathbf{g}_{0,0}+{\frac{G}{r_{12}}}^{(3.5)} \mathbf{g}_{0,1} m_{2}+\frac{G^{2}}{r_{12}^{2}}\left[{ }^{(3.5)} \mathbf{g}_{0,2} m_{2}^{2}+{ }^{(3.5)} \mathbf{g}_{1,1} m_{1} m_{2}\right]+1 \leftrightarrow 2,
$$

$$
\begin{aligned}
{ }^{(3.5)} \mathbf{g}_{0,0} & =-\frac{3}{8} v_{1}^{4} \mathbf{S}_{1} \times \mathbf{v}_{1} \\
{ }^{(3.5)} \mathbf{g}_{0,1} & =\left(n_{12}, S_{1}, v_{1}\right) \mathbf{n}_{12}\left[-\frac{3}{4}\left(n_{12} v_{1}\right)^{2}-\frac{9}{8}\left(n_{12} v_{1}\right)\left(n_{12} v_{2}\right)-\frac{3}{4}\left(n_{12} v_{2}\right)^{2}+\frac{9}{16} v_{1}^{2}-\frac{7}{8} v_{2}^{2}\right] \\
& +\left(n_{12}, S_{1}, v_{2}\right) \mathbf{n}_{12}\left[\frac{3}{4}\left(n_{12} v_{1}\right)^{2}+\frac{3}{2}\left(n_{12} v_{1}\right)\left(n_{12} v_{2}\right)-\frac{3}{4}\left(n_{12} v_{2}\right)^{2}-\frac{3}{8} v_{1}^{2}+\frac{3}{2}\left(v_{1} v_{2}\right)-\frac{1}{4} v_{2}^{2}\right] \\
& +\left(S_{1}, v_{1}, v_{2}\right) \mathbf{n}_{12}\left[\frac{1}{4}\left(n_{12} v_{1}\right)+2\left(n_{12} v_{2}\right)\right]+\left(n_{12}, S_{1}, v_{1}\right) \mathbf{v}_{1}\left[-\left(n_{12} v_{1}\right)-\frac{9}{8}\left(n_{12} v_{2}\right)\right] \\
& +\left(n_{12}, S_{1}, v_{2}\right) \mathbf{v}_{1}\left[\frac{5}{8}\left(n_{12} v_{1}\right)+\frac{1}{2}\left(n_{12} v_{2}\right)\right]+\left(n_{12}, S_{1}, v_{1}\right) \mathbf{v}_{2}\left[\frac{3}{2}\left(n_{12} v_{1}\right)+\frac{5}{2}\left(n_{12} v_{2}\right)\right] \\
& +\left(n_{12}, S_{1}, v_{2}\right) \mathbf{v}_{2}\left[-\frac{3}{2}\left(n_{12} v_{1}\right)-\frac{1}{2}\left(n_{12} v_{2}\right)\right]+\frac{9}{8}\left(S_{1}, v_{1}, v_{2}\right) \mathbf{v}_{1}-\left(S_{1}, v_{1}, v_{2}\right) \mathbf{v}_{2} \\
& +\left(n_{12}, S_{1}, v_{1}\right) \tilde{\mathbf{y}}_{1}\left[-\frac{3}{8}\left(n_{12} v_{1}\right)\left(n_{12} v_{2}\right)-\frac{5}{16} v_{1}^{2}+\frac{1}{8}\left(v_{1} v_{2}\right)-\frac{7}{8} v_{2}^{2}\right]+v_{2}^{2}\left(n_{12}, S_{1}, v_{2}\right) \tilde{\mathbf{y}}_{1} \\
& +\left(n_{12}, S_{1}, v_{1}\right) \tilde{\mathbf{y}}_{2}\left[-\frac{21}{8}\left(n_{12} v_{1}\right)\left(n_{12} v_{2}\right)-\frac{3}{2}\left(n_{12} v_{2}\right)^{2}-\frac{3}{16} v_{1}^{2}-\frac{9}{8}\left(v_{1} v_{2}\right)+\frac{15}{8} v_{2}^{2}\right] \\
& +\left(n_{12}, S_{1}, v_{2}\right) \tilde{\mathbf{y}}_{2}\left[3\left(n_{12} v_{1}\right)^{2}+3\left(n_{12} v_{1}\right)\left(n_{12} v_{2}\right)-v_{1}^{2}+3\left(v_{1} v_{2}\right)-3 v_{2}^{2}\right] \\
& +\left(S_{1}, v_{1}, v_{2}\right) \tilde{\mathbf{y}}_{2}\left[\frac{15}{8}\left(n_{12} v_{1}\right)+2\left(n_{12} v_{2}\right)\right]-\frac{7}{8}\left(n_{12} v_{1}\right)\left(S_{1}, v_{1}, v_{2}\right) \tilde{\mathbf{y}}_{1}
\end{aligned}
$$




$$
\begin{aligned}
&+ \mathbf{S}_{1} \times \mathbf{v}_{1}\left[\frac{1}{2}\left(n_{12} v_{1}\right)^{2}+\frac{5}{8}\left(n_{12} v_{1}\right)\left(n_{12} v_{2}\right)-3 v_{1}^{2}+\frac{23}{8}\left(v_{1} v_{2}\right)-v_{2}^{2}\right] \\
&+ \mathbf{S}_{1} \times \mathbf{v}_{2}\left[-\frac{1}{8}\left(n_{12} v_{1}\right)^{2}+\left(n_{12} v_{1}\right)\left(n_{12} v_{2}\right)-\left(n_{12} v_{2}\right)^{2}+\frac{17}{8} v_{1}^{2}-3\left(v_{1} v_{2}\right)+2 v_{2}^{2}\right] \\
&+ \mathbf{n}_{12} \times \mathbf{S}_{1}\left[\frac{3}{4}\left(n_{12} v_{1}\right)^{3}+\frac{3}{4}\left(n_{12} v_{1}\right)^{2}\left(n_{12} v_{2}\right)+\frac{3}{4}\left(n_{12} v_{1}\right)\left(n_{12} v_{2}\right)^{2}+\frac{3}{4}\left(n_{12} v_{2}\right)^{3}\right. \\
&-\frac{3}{4}\left(n_{12} v_{1}\right) v_{1}^{2}-\frac{1}{8}\left(n_{12} v_{2}\right) v_{1}^{2}+\frac{3}{8}\left(n_{12} v_{1}\right)\left(v_{1} v_{2}\right)-\frac{1}{2}\left(n_{12} v_{2}\right)\left(v_{1} v_{2}\right)+\frac{1}{4}\left(n_{12} v_{1}\right) v_{2}^{2} \\
&\left.-\frac{5}{4}\left(n_{12} v_{2}\right) v_{2}^{2}\right], \\
&=-\frac{39}{4}\left(n_{12}, S_{1}, v_{1}\right) \mathbf{n}_{12}+13\left(n_{12}, S_{1}, v_{2}\right) \mathbf{n}_{12}-\frac{9}{4}\left(n_{12}, S_{1}, v_{1}\right) \tilde{\mathbf{y}}_{1}+\left(n_{12}, S_{1}, v_{2}\right) \tilde{\mathbf{y}}_{1} \\
&+ \frac{17}{4}\left(n_{12}, S_{1}, v_{1}\right) \tilde{\mathbf{y}}_{2}-\left(n_{12}, S_{1}, v_{2}\right) \tilde{\mathbf{y}}_{2}+10 \mathbf{S}_{1} \times \mathbf{v}_{1}-10 \mathbf{S}_{1} \times \mathbf{v}_{2} \\
&+ \mathbf{n}_{12} \times \mathbf{S}_{1}\left[\frac{29}{2}\left(n_{12} v_{1}\right)-\frac{23}{2}\left(n_{12} v_{2}\right)\right], \\
&\left.\mathbf{g}_{1,1}\right] \frac{55}{8}\left(n_{12}, S_{1}, v_{1}\right) \mathbf{n}_{12}-\frac{19}{4}\left(n_{12}, S_{1}, v_{2}\right) \mathbf{n}_{12}+\frac{1}{2}\left(n_{12}, S_{1}, v_{1}\right) \tilde{\mathbf{y}}_{1}-\frac{25}{8}\left(n_{12}, S_{1}, v_{2}\right) \tilde{\mathbf{y}}_{1} \\
&- \frac{1}{2}\left(n_{12}, S_{1}, v_{1}\right) \tilde{\mathbf{y}}_{2}+\frac{49}{8}\left(n_{12}, S_{1}, v_{2}\right) \tilde{\mathbf{y}}_{2}-\frac{45}{8} \mathbf{S}_{1} \times \mathbf{v}_{1}+\frac{45}{8} \mathbf{S}_{1} \times \mathbf{v}_{2} \\
&+ \mathbf{n}_{12} \times \mathbf{S}_{1}\left[-\frac{59}{8}\left(n_{12} v_{1}\right)+\frac{87}{8}\left(n_{12} v_{2}\right)\right] \cdot \\
& \mathbf{g}_{0,2}
\end{aligned}
$$

This result translates into a next-to-next-to-leading order correction to the vector $\mathbf{z}$ in Eqs. (3.3), of the form

$$
\delta \mathbf{z}=\frac{1}{c^{7}}\left[{ }^{(3.5)} \mathbf{z}^{(0)}+\frac{G m}{r}^{(3.5)} \mathbf{z}^{(1)}+{\frac{G^{2} m^{2}}{r^{2}}}^{(3.5)} \mathbf{z}^{(2)}\right]
$$

with

$$
\begin{aligned}
{ }^{(3.5)} \mathbf{z}^{(0)} & =\boldsymbol{\Sigma} \times \mathbf{v}\left(-\frac{3}{8}+\frac{13}{4} \nu-7 \nu^{2}\right) v^{4} \\
{ }^{(3.5)} \mathbf{z}^{(1)} & =(n, \Sigma, v) \mathbf{n}\left[\left(-\frac{3}{4}+\frac{9}{2} \nu-6 \nu^{2}\right)(n v)^{2}+\left(\frac{1}{4}+\nu-8 \nu^{2}\right) v^{2}\right] \\
& +(n, S, v) \mathbf{n}\left[\frac{\delta m}{m}\left(-\frac{3}{2}+\frac{9}{2} \nu\right)(n v)^{2}+\frac{\delta m}{m}(3-\nu) v^{2}\right] \\
& +(n, \Sigma, v) \mathbf{v}\left(-1+\frac{7}{2} \nu+2 \nu^{2}\right)(n v)+(n, S, v) \mathbf{v} \frac{\delta m}{m}\left(-\frac{3}{2}-\nu\right)(n v) \\
& +\mathbf{n} \times \mathbf{S}\left[\frac{\delta m}{m}\left(\frac{9}{8}-\frac{9}{4} \nu\right)(n v)^{3}+\frac{\delta m}{m}\left(-\frac{7}{8}+4 \nu\right)(n v) v^{2}\right] \\
& +\mathbf{n} \times \mathbf{\Sigma}\left[\left(\frac{3}{4}-\frac{9}{2} \nu+6 \nu^{2}\right)(n v)^{3}+\left(-\frac{3}{4}+\frac{23}{4} \nu-11 \nu^{2}\right)(n v) v^{2}\right]
\end{aligned}
$$




$$
\begin{aligned}
+ & \mathbf{S} \times \mathbf{v}\left[\frac{\delta m}{m}\left(\frac{9}{8}-\frac{3}{4} \nu\right)(n v)^{2}+\frac{\delta m}{m}\left(-\frac{3}{8}+\nu\right) v^{2}\right] \\
& +\boldsymbol{\Sigma} \times \mathbf{v}\left[\left(\frac{1}{2}-2 \nu+\frac{9}{2} \nu^{2}\right)(n v)^{2}+\left(-3+\frac{25}{4} \nu+6 \nu^{2}\right) v^{2}\right], \\
{ }^{(3.5)} \mathbf{z}^{(2)} & =(n, \Sigma, v) \mathbf{n}\left(\frac{59}{8}-\frac{161}{4} \nu+6 \nu^{2}\right)+\frac{171}{8} \frac{\delta m}{m}(n, S, v) \mathbf{n} \\
& +\mathbf{n} \times \mathbf{S} \frac{\delta m}{m}\left(-\frac{133}{8}-4 \nu\right)(n v)+\mathbf{n} \times \mathbf{\Sigma}\left(-\frac{59}{8}+\frac{137}{4} \nu+12 \nu^{2}\right)(n v) \\
& +\mathbf{S} \times \mathbf{v} \frac{\delta m}{m}\left(-\frac{97}{8}-\nu\right)+\mathbf{\Sigma} \times \mathbf{v}\left(-\frac{45}{8}+\frac{119}{4} \nu-\nu^{2}\right) .
\end{aligned}
$$

To conclude this Appendix, let us show explicitly that the above $\mathcal{O}\left(1 / c^{7}\right)$ correction to $\mathbf{y}_{1}$ written in terms of the CM variables cancels out when reducing our general frame results to the center-of-mass frame. Let us denote by $\mathbf{z}$ the $\mathcal{O}\left(1 / c^{2}\right)$ correction to the Newtonian expressions of $\mathbf{y}_{1}$ and $\mathbf{y}_{2}$, as defined by Eqs. (3.3). Since we want to obtain expressions up to $\mathcal{O}\left(1 / c^{7}\right)$ in the $\mathrm{CM}$ frame, the terms of order $\mathcal{O}\left(1 / c^{7}\right)$ in $\mathbf{z}$ can only play a role in the reduction of the Newtonian parts. These Newtonian contributions are very simple, so we can check that the expression of $\mathbf{z}$ is needed only up to $\mathcal{O}\left(1 / c^{5}\right)$.

The simplest case is that of the relative Newtonian acceleration $\mathbf{a}_{\mathrm{N}}=-G m \mathbf{n}_{\mathbf{1 2}} / r_{12}^{2}$, which receives no higher order correction when reexpressing it in the $\mathrm{CM}$ frame. The same argument applies to the near zone metric. By contrast, the Newtonian energy

$$
E_{\mathrm{N}}=\frac{1}{2} m \nu v^{2}+m\left(\frac{\mathrm{d} \mathbf{z}}{\mathrm{d} t}\right)^{2}-\frac{G m \nu}{2 r},
$$

receives a PN correction when expressed in terms of the $\mathrm{CM}$ quantities. However, since the terms linear in $\mathbf{z}$ cancel out while $\mathrm{d} \mathbf{z} / \mathrm{d} t=\mathcal{O}\left(1 / c^{2}\right)$, we only need $\mathbf{z}$ up to the order $\mathcal{O}\left(1 / c^{5}\right)$ to get the energy up to the $3.5 \mathrm{PN}$ level. A similar argument applies to the Newtonian angular momentum, which is deprived of linear-in-z contributions:

$$
\mathbf{L}_{\mathrm{N}}=m \nu \mathbf{x} \times \mathbf{v}+m \mathbf{z} \times \frac{\mathrm{d} \mathbf{z}}{\mathrm{d} t} .
$$

\section{Appendix D: Orbital angular momentum}

The conserved total angular momentum (such that $\mathrm{d} \mathbf{J} / \mathrm{d} t=0$ ) is conventionaly split into an orbital angular momentum, which will also receive contributions from the spins, and the sum of spins of the system:

$$
\mathbf{J}=\mathbf{L}+\frac{\mathbf{S}}{c} .
$$

This actually represents the definition of the orbital angular momentum $\mathbf{L}$, which depends on the choice of variables for the individual spins. With this definition, the structure of the orbital part is

$$
\mathbf{L}=\nu\left\{\boldsymbol{\ell}_{\mathrm{N}}+\frac{1}{c^{2}} \boldsymbol{\ell}_{1 \mathrm{PN}}+\frac{1}{c^{3}} \boldsymbol{\ell}_{S} 1.5 \mathrm{PN}+\frac{1}{c^{4}}\left[\boldsymbol{\ell}_{2 \mathrm{PN}}+\underset{S S}{\boldsymbol{\ell}_{2} \mathrm{PN}}\right]+\frac{1}{c^{5}} \boldsymbol{\ell}_{S} 2.5 \mathrm{PN}\right.
$$




$$
\left.+\frac{1}{c^{6}}\left[\ell_{3 \mathrm{PN}}+\underset{S S}{\ell} 3 \mathrm{PN}\right]+\frac{1}{c^{7}} \ell_{S} 3.5 \mathrm{PN}+\mathcal{O}\left(\frac{1}{c^{8}}\right)\right\} .
$$

The non-spin terms up to 3PN order can be found in Ref. [53]. Again neglecting the spin-spin terms, we arrive at the following spin-orbit contributions. At leading and next-to-leading order, we have

$$
\begin{aligned}
& \boldsymbol{\ell}_{S} 1.5 \mathrm{PN}={ }^{(1.5)} \boldsymbol{\ell}_{0}+{ }^{(1.5)} \boldsymbol{\ell}_{1} \frac{G m}{r} \\
& { }^{(1.5)} \boldsymbol{\ell}_{0}=-\frac{1}{2} \frac{\delta m}{m}(\Sigma v) \mathbf{v}-\frac{1}{2}(S v) \mathbf{v}+\frac{1}{2} v^{2} \mathbf{S}+\frac{1}{2} \frac{\delta m}{m} v^{2} \mathbf{\Sigma} \\
& { }^{(1.5)} \boldsymbol{\ell}_{1}=\frac{\delta m}{m}(n \Sigma) \mathbf{n}+3(n S) \mathbf{n}-3 \mathbf{S}-\frac{\delta m}{m} \mathbf{\Sigma},
\end{aligned}
$$

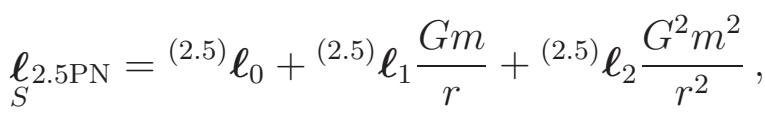

$$
\begin{aligned}
& { }^{(2.5)} \boldsymbol{\ell}_{0}=(\Sigma v) \mathbf{v} \frac{\delta m}{m}\left(-\frac{3}{8}+\frac{5}{4} \nu\right) v^{2}+(S v) \mathbf{v}\left(-\frac{3}{8}+\frac{9}{8} \nu\right) v^{2}+\mathbf{S}\left(\frac{3}{8}-\frac{9}{8} \nu\right) v^{4} \\
& +\Sigma \frac{\delta m}{m}\left(\frac{3}{8}-\frac{5}{4} \nu\right) v^{4} \\
& { }^{(2.5)} \boldsymbol{\ell}_{1}=(n \Sigma) \mathbf{n}\left[-3 \nu \frac{\delta m}{m}(n v)^{2}+\frac{\delta m}{m}\left(\frac{1}{2}+\frac{3}{2} \nu\right) v^{2}\right]+(\Sigma v) \mathbf{n} \frac{\delta m}{m}\left(-\frac{1}{2}-\frac{13}{4} \nu\right)(n v) \\
& +(n S) \mathbf{n}\left[-\frac{9}{2} \nu(n v)^{2}+\left(\frac{7}{2}-\frac{1}{2} \nu\right) v^{2}\right]+(S v) \mathbf{n}\left(-\frac{3}{2}-\frac{7}{2} \nu\right)(n v) \\
& +\frac{7}{4} \nu \frac{\delta m}{m}(n v)(n \Sigma) \mathbf{v}+(\Sigma v) \mathbf{v} \frac{\delta m}{m}\left(-\frac{3}{2}+\frac{1}{2} \nu\right)+(n S) \mathbf{v}(-3+6 \nu)(n v) \\
& +(S v) \mathbf{v}\left(\frac{1}{2}-\frac{1}{2} \nu\right)+\mathbf{S}\left[\left(2+\frac{5}{2} \nu\right)(n v)^{2}+\left(-\frac{3}{2}+\frac{1}{2} \nu\right) v^{2}\right] \\
& +\Sigma\left[\frac{7}{2} \nu \frac{\delta m}{m}(n v)^{2}+\frac{\delta m}{m}\left(\frac{3}{2}-\nu\right) v^{2}\right] \text {, } \\
& { }^{(2.5)} \boldsymbol{\ell}_{2}=(n \Sigma) \mathbf{n} \frac{\delta m}{m}\left(-\frac{1}{2}-\frac{3}{2} \nu\right)+(n S) \mathbf{n}\left(-\frac{1}{2}-2 \nu\right)+\mathbf{S}\left(\frac{1}{2}+2 \nu\right) \\
& +\Sigma \frac{\delta m}{m}\left(\frac{1}{2}+\frac{3}{2} \nu\right) \text {. }
\end{aligned}
$$

As in the case for the energy, the next-to-leading term does not have the same expression as the one published for instance in Refs. [23, 28], due to our different choice of spin variables; but now the difference also shows up in the leading-order spin contributions to $\mathbf{L}$ since it is actually the next-to-leading order correction in the total angular momentum $\mathbf{J}$. Finally, at next-to-next-to-leading order we have

$$
{ }_{S} \ell_{3.5 \mathrm{PN}}={ }^{(3.5)} \boldsymbol{\ell}_{0}+{ }^{(3.5)} \boldsymbol{\ell}_{1} \frac{G m}{r}+{ }^{(3.5)} \boldsymbol{\ell}_{2} \frac{G^{2} m^{2}}{r^{2}}+{ }^{(3.5)} \boldsymbol{\ell}_{3} \frac{G^{3} m^{3}}{r^{3}},
$$




$$
\begin{aligned}
& { }^{(3.5)} \boldsymbol{\ell}_{0}=(\Sigma v) \mathbf{v} \frac{\delta m}{m}\left(-\frac{5}{16}+\frac{19}{8} \nu-\frac{75}{16} \nu^{2}\right) v^{4}+(S v) \mathbf{v}\left(-\frac{5}{16}+\frac{35}{16} \nu-\frac{65}{16} \nu^{2}\right) v^{4} \\
& +\mathbf{S}\left(\frac{5}{16}-\frac{35}{16} \nu+\frac{65}{16} \nu^{2}\right) v^{6}+\Sigma \frac{\delta m}{m}\left(\frac{5}{16}-\frac{19}{8} \nu+\frac{75}{16} \nu^{2}\right) v^{6}, \\
& { }^{(3.5)} \boldsymbol{\ell}_{1}=(n \Sigma) \mathbf{n}\left[\frac{\delta m}{m}\left(\frac{15}{4} \nu-\frac{75}{8} \nu^{2}\right)(n v)^{4}+\frac{\delta m}{m}\left(-\frac{15}{2} \nu+9 \nu^{2}\right)(n v)^{2} v^{2}\right. \\
& \left.+\frac{\delta m}{m}\left(\frac{3}{8}+\frac{27}{8} \nu-\frac{77}{8} \nu^{2}\right) v^{4}\right]+(\Sigma v) \mathbf{n}\left[\frac{\delta m}{m}\left(\frac{51}{16} \nu+\frac{15}{8} \nu^{2}\right)(n v)^{3}\right. \\
& \left.+\frac{\delta m}{m}\left(-\frac{3}{8}-\frac{23}{4} \nu+\frac{243}{16} \nu^{2}\right)(n v) v^{2}\right]+(n S) \mathbf{n}\left[\left(\frac{45}{8} \nu-\frac{135}{8} \nu^{2}\right)(n v)^{4}\right. \\
& \left.+\left(-\frac{33}{4} \nu+\frac{33}{4} \nu^{2}\right)(n v)^{2} v^{2}+\left(\frac{33}{8}-\frac{83}{8} \nu-\frac{11}{8} \nu^{2}\right) v^{4}\right] \\
& +(S v) \mathbf{n}\left[\left(-\frac{3}{4}+\frac{9}{2} \nu+\frac{33}{4} \nu^{2}\right)(n v)^{3}+\left(-\frac{17}{8}+\frac{5}{16} \nu+\frac{109}{8} \nu^{2}\right)(n v) v^{2}\right] \\
& +(n \Sigma) \mathbf{v}\left[\frac{\delta m}{m}\left(-\frac{21}{16} \nu+\frac{93}{8} \nu^{2}\right)(n v)^{3}+\frac{\delta m}{m}\left(\frac{1}{4} \nu-\frac{31}{16} \nu^{2}\right)(n v) v^{2}\right] \\
& +(\Sigma v) \mathbf{v}\left[\frac{\delta m}{m}\left(-\frac{3}{4} \nu-\frac{19}{8} \nu^{2}\right)(n v)^{2}+\frac{\delta m}{m}\left(-\frac{21}{8}+\frac{59}{8} \nu-\frac{35}{8} \nu^{2}\right) v^{2}\right] \\
& +(n S) \mathbf{v}\left[\left(\frac{3}{4}-\frac{15}{2} \nu+27 \nu^{2}\right)(n v)^{3}+\left(-\frac{17}{4}+\frac{295}{16} \nu-\frac{75}{4} \nu^{2}\right)(n v) v^{2}\right] \\
& +(S v) \mathbf{v}\left[\left(\frac{1}{8} \nu-\frac{43}{4} \nu^{2}\right)(n v)^{2}+\left(\frac{3}{8}-\frac{31}{8} \nu+\frac{5}{8} \nu^{2}\right) v^{2}\right] \\
& +\mathbf{S}\left[\left(-\frac{21}{8} \nu-\frac{33}{8} \nu^{2}\right)(n v)^{4}+\left(3-\frac{9}{4} \nu-\frac{21}{4} \nu^{2}\right)(n v)^{2} v^{2}+\left(-\frac{9}{8}+\frac{47}{8} \nu-\frac{5}{8} \nu^{2}\right) v^{4}\right] \\
& +\boldsymbol{\Sigma}\left[\frac{\delta m}{m}\left(-\frac{33}{8} \nu+\frac{9}{8} \nu^{2}\right)(n v)^{4}+\frac{\delta m}{m}\left(\frac{39}{4} \nu-17 \nu^{2}\right)(n v)^{2} v^{2}\right. \\
& \left.+\frac{\delta m}{m}\left(\frac{21}{8}-\frac{33}{4} \nu+\frac{47}{8} \nu^{2}\right) v^{4}\right] \text {, } \\
& { }^{(3.5)} \boldsymbol{\ell}_{2}=(n \Sigma) \mathbf{n}\left[\frac{\delta m}{m}\left(-\frac{469}{8} \nu-\frac{51}{4} \nu^{2}\right)(n v)^{2}+\frac{\delta m}{m}\left(\frac{7}{4}-\frac{149}{4} \nu+\frac{33}{4} \nu^{2}\right) v^{2}\right] \\
& +(n S) \mathbf{n}\left[\left(-\frac{973}{8} \nu-\frac{39}{2} \nu^{2}\right)(n v)^{2}+\left(\frac{55}{4}-\frac{311}{4} \nu+6 \nu^{2}\right) v^{2}\right] \\
& +(\Sigma v) \mathbf{n} \frac{\delta m}{m}\left(-\frac{17}{8}+\frac{103}{4} \nu-\frac{25}{2} \nu^{2}\right)(n v)+(S v) \mathbf{n}\left(-\frac{29}{8}+\frac{247}{4} \nu-\frac{31}{2} \nu^{2}\right)(n v) \\
& +(n \Sigma) \mathbf{v} \frac{\delta m}{m}\left(-\frac{1}{8}+\frac{177}{2} \nu+3 \nu^{2}\right)(n v)+(\Sigma v) \mathbf{v} \frac{\delta m}{m}\left(-\frac{7}{4}-\frac{257}{8} \nu+\frac{21}{4} \nu^{2}\right) \\
& +(n S) \mathbf{v}\left(-\frac{117}{8}+\frac{731}{4} \nu+\frac{27}{2} \nu^{2}\right)(n v)+(S v) \mathbf{v}\left(\frac{25}{4}-\frac{571}{8} \nu+\frac{9}{2} \nu^{2}\right) \\
& +\mathbf{S}\left[\left(\frac{17}{2}-\frac{163}{8} \nu+\frac{35}{2} \nu^{2}\right)(n v)^{2}+\left(-\frac{41}{4}+\frac{373}{8} \nu-\frac{13}{2} \nu^{2}\right) v^{2}\right]
\end{aligned}
$$




$$
\begin{aligned}
& +\boldsymbol{\Sigma}\left[\frac{\delta m}{m}\left(\frac{1}{2}-\frac{43}{8} \nu+\frac{33}{2} \nu^{2}\right)(n v)^{2}+\frac{\delta m}{m}\left(\frac{7}{4}+\frac{153}{8} \nu-\frac{31}{4} \nu^{2}\right) v^{2}\right] \\
{ }^{(3.5)} \boldsymbol{\ell}_{3} & =(n \Sigma) \mathbf{n} \frac{\delta m}{m}\left(\frac{1}{2}-2 \nu-\frac{3}{2} \nu^{2}\right)+(n S) \mathbf{n}\left(\frac{3}{2}-\frac{13}{4} \nu-2 \nu^{2}\right)+\mathbf{S}\left(-\frac{3}{2}+\frac{13}{4} \nu+2 \nu^{2}\right) \\
+ & \boldsymbol{\Sigma} \frac{\delta m}{m}\left(-\frac{1}{2}+2 \nu+\frac{3}{2} \nu^{2}\right) .
\end{aligned}
$$

\section{Appendix E: Gauging away the 3PN spin-orbit terms}

The metric regularized at the location of the two particles, as was presented in Section IIIE, displays terms which are formally of $3 \mathrm{PN}$ order, that is to say of order $\mathcal{O}\left(1 / c^{8}\right)$ in $g_{00}$ and $\mathcal{O}\left(1 / c^{7}\right)$ in $g_{0 i}$. These terms are given by the expressions (3.11c) and (3.12C):

$$
\begin{aligned}
& \left(\begin{array}{l}
g_{S}^{3 \mathrm{PN}} \\
S^{00}
\end{array}\right)_{1}=\frac{G^{2} m \nu}{c^{8} r^{3}}\left\{(n, \Sigma, v)\left(2-10 \frac{\delta m}{m}\right)(n v)-24(n v)(n, S, v)\right\}, \\
& \left(g_{S}^{3 \mathrm{PN}}\right)_{1}=\frac{G^{2} m \nu}{c^{7} r^{3}}\left\{2(n v)(\mathbf{n} \times \mathbf{\Sigma})^{i}-\frac{2}{3}(\mathbf{v} \times \mathbf{\Sigma})^{i}\right\} .
\end{aligned}
$$

In this Appendix, we show that they are actually a pure coordinate effect, in the sense that they can be eliminated by a gauge transformation. We already encountered such a gauge transformation in Ref. [51] and in Paper I (see the footnote in Section VI B there), where it was used to remove the $\mathcal{O}\left(1 / c^{6}\right)$ terms appearing in the acceleration. Here we generalize this transformation to cancel as well the $3 \mathrm{PN}$ terms in the components of the regularized metric. Notice that, for $g_{00}$ and $g_{0 i}$, we do not control the metric components in the bulk at the corresponding order. The transformation we present here is only suitable for removing these terms in the regularized metric.

We consider a coordinate transformation $x^{\prime \mu}=x^{\mu}+\xi^{\mu}$, which has to satisfy $\square \xi^{\mu}=0$ (at dominant order) to respect the harmonic gauge condition. We will need to have $\xi^{0}=\mathcal{O}\left(1 / c^{7}\right)$ and $\xi^{i}=\mathcal{O}\left(1 / c^{6}\right)$. Since the components $g_{i j}$ of the metric must remain unaffected in the bulk at order $\mathcal{O}\left(1 / c^{6}\right)$ (see (3.16) ), we further require that the component $\xi^{i}$ is a mere function of time, i.e. does not depend on the field point $\mathbf{x}$. We allow a spatial dependence in the component $\xi^{0}$, but the harmonicity condition $\Delta \xi^{0}=\mathcal{O}\left(1 / c^{9}\right)$ constrains its structure. Hence, we take the following Ansatz for the transformation:

$$
\begin{aligned}
\xi^{0} & =\frac{G m^{2} \nu}{c^{7}}\left[A+B_{1} \frac{1}{r_{1}}+B_{2} \frac{1}{r_{2}}+C_{1}^{i} \partial_{i}\left(\frac{1}{r_{1}}\right)+C_{2}^{i} \partial_{i}\left(\frac{1}{r_{2}}\right)\right], \\
\xi^{i} & =\frac{G^{2} m \nu}{c^{6}} D^{i},
\end{aligned}
$$

where $A, B_{1}, B_{2}, C_{1}^{i}, C_{2}^{i}$ and $D^{i}$ are all mere functions of time. This gauge transformation has to be symmetric by exchange of the two bodies, which relates $B_{1}$ to $B_{2}, C_{1}^{i}$ to $C_{2}^{i}$ and constrains the expression of $A$ and $D^{i}$. We could have considered higher order derivatives of $1 / r$ in $\xi^{0}$, but we found that the above structure was sufficient for our purpose.

With our simple choice for $\xi^{i}$, the gauge transformation changes the metric components according to, (see Eqs (6.9) in [48]):

$$
\delta_{\xi} g_{00}=2 \partial_{0} \xi^{0}+\mathcal{O}\left(1 / c^{10}\right),
$$




$$
\begin{aligned}
& \delta_{\xi} g_{0 i}=-\partial_{0} \xi^{i}+\partial_{i} \xi^{0}+\mathcal{O}\left(1 / c^{9}\right), \\
& \delta_{\xi} g_{i j}=-\partial_{i} \xi^{j}-\partial_{j} \xi^{i}+\mathcal{O}\left(1 / c^{8}\right),
\end{aligned}
$$

while the acceleration transforms as (see Eqs. (4.8) in [54 for more general expressions):

$$
\delta_{\xi} a_{1}^{i}=\frac{\mathrm{d}^{2}}{\mathrm{~d} t^{2}} \xi^{i}(t)+\mathcal{O}\left(1 / c^{8}\right)
$$

This shows that $\xi^{i}$ is the same as we used in Paper I, and which was called $\delta X^{i}$ there. Solving for the other unknown functions of time by applying (E4) to the structure (E3), we find the following expressions:

$$
\begin{aligned}
A & =\frac{1}{r^{2}}\left[4(\mathbf{n}, \mathbf{v}, \mathbf{S})+\frac{3}{2} \frac{\delta m}{m}(\mathbf{n}, \mathbf{v}, \mathbf{\Sigma})\right], \\
B_{2} & =-B_{1}=-\frac{2}{3 r}(\mathbf{n}, \mathbf{v}, \boldsymbol{\Sigma}), \\
\mathbf{C}_{2} & =\mathbf{C}_{1}=-\frac{5}{6}(\mathbf{n}, \mathbf{v}, \boldsymbol{\Sigma}) \mathbf{n}+\frac{1}{3} \mathbf{v} \times \mathbf{\Sigma}-(n v) \mathbf{n} \times \mathbf{\Sigma}, \\
\mathbf{D} & =-\frac{1}{r^{2}} \mathbf{n} \times \mathbf{\Sigma} .
\end{aligned}
$$

This solution allows us, within the class of harmonic coordinates, to cancel the 3PN terms (E1) in the regularized metric, which are therefore pure gauge.

[1] B. Abbott et al. (LIGO Scientific Collaboration), Rept.Prog.Phys. 72, 076901 (2009), arXiv:0711.3041 [gr-qc].

[2] LIGO's website, http://www.ligo.caltech.edu/.

[3] T. Accadia et al. (VIRGO Collaboration), JINST 7, P03012 (2012).

[4] Virgo's website, https://wwwcascina.virgo.infn.it/.

[5] H. Grote (LIGO Scientific Collaboration), Class.Quant.Grav. 25, 114043 (2008).

[6] K. Kuroda (LCGT Collaboration), Class.Quant.Grav. 27, 084004 (2010).

[7] P. Amaro-Seoane, S. Aoudia, S. Babak, P. Binetruy, E. Berti, et al., (2012), arXiv:1201.3621 [astro-ph.CO].

[8] eLISA's website, Http://www.elisa-ngo.org/.

[9] C. Cutler, T. A. Apostolatos, L. Bildsten, L. S. Finn, E. E. Flanagan, et al., Phys.Rev.Lett. 70, 2984 (1993), arXiv:9208005 [astro-ph],

[10] C. Cutler and E. E. Flanagan, Phys.Rev. D49, 2658 (1994), arXiv:9402014 [gr-qc],

[11] L. Blanchet, Living Rev.Rel. 9, 4 (2006).

[12] L. Blanchet, T. Damour, G. Esposito-Farèse, and B. R. Iyer, Phys.Rev.Lett. 93, 091101 (2004), arXiv:0406012 [gr-qc],

[13] K. Arun, L. Blanchet, B. R. Iyer, and M. S. Qusailah, Class.Quant.Grav. 21, 3771 (2004), arXiv:0404085 [gr-qc],

[14] L. Blanchet, G. Faye, B. R. Iyer, and B. Joguet, Phys.Rev. D65, 061501 (2002), arXiv:0105099 [gr-qc]

[15] M. A. Abramowicz and W. Kluzniak, Astron. Astrophys. 374, L19 (2001), arXiv:0105077 [astro-ph]. 
[16] T. E. Strohmayer, ApJ 552, L49 (2001), arXiv:0104487 [astro-ph].

[17] J. E. McClintock, R. Shafee, R. Narayan, R. A. Remillard, S. W. Davis, et al., Astrophys.J. 652, 518 (2006), arXiv:0606076 [astro-ph],

[18] L. Gou, J. E. McClintock, M. J. Reid, J. A. Orosz, J. F. Steiner, et al., Astrophys.J. 742, 85 (2011), arXiv:1106.3690 [astro-ph.HE],

[19] M. A. Nowak, J. Wilms, K. Pottschmidt, N. Schulz, D. Maitra, et al., Astrophys.J. 744, 107 (2012), arXiv:1109.6008 [astro-ph.HE],

[20] B. Barker and R. O'Connell, Phys.Rev. D12, 329 (1975).

[21] B. M. Barker and R. F. O'Connell, Gen. Rel. and Grav. 11, 149 (1979).

[22] L. Kidder, C. Will, and A. Wiseman, Phys. Rev. D 47, R4183 (1993).

[23] L. E. Kidder, Phys.Rev. D52, 821 (1995), arXiv:9506022 [gr-qc],

[24] W. D. Goldberger and I. Z. Rothstein, Phys.Rev. D73, 104029 (2006), arXiv:0409156 [hep-th],

[25] R. A. Porto, Phys.Rev. D73, 104031 (2006), arXiv:0511061 [gr-qc],

[26] B. J. Owen, H. Tagoshi, and A. Ohashi, Phys.Rev. D57, 6168 (1998), arXiv:9710134 [gr-qc].

[27] H. Tagoshi, A. Ohashi, and B. J. Owen, Phys.Rev. D63, 044006 (2001) arXiv:0010014 [gr-qc],

[28] G. Faye, L. Blanchet, and A. Buonanno, Phys.Rev. D74, 104033 (2006), arXiv:0605139 [gr-qc].

[29] T. Damour, P. Jaranowski, and G. Schaefer, Phys.Rev. D77, 064032 (2008), arXiv:0711.1048 [gr-qc],

[30] M. Levi, Phys.Rev. D82, 104004 (2010), arXiv:1006.4139 [gr-qc],

[31] R. A. Porto, Class.Quant.Grav. 27, 205001 (2010), arXiv:1005.5730 [gr-qc],

[32] S. Hergt, J. Steinhoff, and G. Schaefer, Class.Quant.Grav. 27, 135007 (2010), arXiv:1002.2093 [gr-qc],

[33] J. Steinhoff, S. Hergt, and G. Schaefer, Phys.Rev. D77, 081501 (2008), arXiv:0712.1716 [gr-qc],

[34] J. Hartung and J. Steinhoff, Phys.Rev. D83, 044008 (2011), arXiv:1011.1179 [gr-qc],

[35] R. A. Porto and I. Z. Rothstein, Phys.Rev. 78, 044012 (2008), arXiv:0802.0720 [gr-qc],

[36] R. A. Porto and I. Z. Rothstein, Phys.Rev. 78, 044013 (2008), arXiv:0804.0260 [gr-qc].

[37] M. Levi, Phys.Rev. D85, 064043 (2012), arXiv:1107.4322 [gr-qc],

[38] J. Hartung and J. Steinhoff, Annalen Phys. 523, 919 (2011), arXiv:1107.4294 [gr-qc].

[39] S. Marsat, A. Bohe, G. Faye, and L. Blanchet, (2012), arXiv:1210.4143 [gr-qc].

[40] J. Hartung and J. Steinhoff, Annalen Phys. 523, 783 (2011), arXiv:1104.3079 [gr-qc].

[41] L. Gallouin, H. Nakano, N. Yunes, and M. Campanelli, "Asymptotically Matched Spacetime Metric for Non-Precessing, Spinning Black Hole Binaries," (2012), to appear in Class. Quant. Grav., arXiv:1208.6489 [gr-qc].

[42] L. Blanchet, A. Buonanno, and A. Le Tiec, to appear in Phys. Rev. D (2012) arXiv:1211.1060 [gr-qc],

[43] J. Friedman, A. Le Tiec, and A. Shab, "unpublished," (2012).

[44] J. M. Martín-García, A. García-Parrado, A. Stecchina, B. Wardell, C. Pitrou, D. Brizuela, D. Yllanes, G. Faye, L. Stein, R. Portugal, and T. Bäckdahl, "xAct: Efficient tensor computer algebra for Mathematica," (GPL 2002-2013), http://www.xact.es/.

[45] C. Will, Phys. Rev. D 71, 084027 (2005), gr-qc/0502039.

[46] L. Blanchet, A. Buonanno, and G. Faye, Phys.Rev. D74, 104034 (2006), erratum Phys.Rev. D 75:049903, 2007, arXiv:0605140 [gr-qc]. 
[47] L. Blanchet and G. Faye, J.Math.Phys. 41, 7675 (2000), arXiv:0004008 [gr-qc],

[48] L. Blanchet and G. Faye, Phys.Rev. D63, 062005 (2001), arXiv:0007051 [gr-qc].

[49] L. Blanchet, T. Damour, and G. Esposito-Farèse, Phys.Rev. D69, 124007 (2004), arXiv:0311052 [gr-qc],

[50] L. Blanchet, G. Faye, and B. Ponsot, Phys.Rev. D58, 124002 (1998), arXiv:9804079 [gr-qc],

[51] L. Blanchet, A. Buonanno, and G. Faye, Phys.Rev. D84, 064041 (2011), arXiv:1104.5659 [gr-qc],

[52] A. Le Tiec, L. Blanchet, and B. F. Whiting, Phys.Rev. D85, 064039 (2012), arXiv:1111.5378 [gr-qc],

[53] L. Blanchet and B. R. Iyer, Class.Quant.Grav. 20, 755 (2003), arXiv:0209089 [gr-qc]

[54] K. G. Arun, L. Blanchet, B. R. Iyer, and M. S. S. Qusailah, Phys. Rev. D 77, 064035 (2008), arXiv:0711.0302 [gr-qc], 